OPEN ACCESS

Edited by:

Alex Shimura Yamashita,

The Johns Hopkins Hospital, Johns

Hopkins Medicine, United States

Reviewed by:

Maria Ana Duhagon,

Universidad de la República, Uruguay

Yanli Li,

Shanghai University, China

*Correspondence.

Yingjie Wu

yingjiewu@dmu.edu.cn

Qi Wang

wqdlmu@163.com

Lin Huang

Ihuang@dmu.edu.cn

tThese authors have contributed equally to this work

Specialty section:

This article was submitted to Molecular and Cellular Oncology, a section of the journal Frontiers in Cell and Developmental Biology

Received: 13 March 2020

Accepted: 07 August 2020 Published: 02 September 2020

Citation:

Liu X, Liu B, Li R, Wang F, Wang $N$, Zhang M, Bai Y, Wu J, Liu L, Han D, Li Z, Feng B, Zhou G, Wang S,

Zeng L, Miao J, Yao Y, Liang B,

Huang L, Wang Q and Wu Y (2020)

miR-146a-5p Plays an Oncogenic

Role in NSCLC via Suppression of TRAF6. Front. Cell Dev. Biol. 8:847.

doi: 10.3389/fcell.2020.00847

\section{miR-146a-5p Plays an Oncogenic Role in NSCLC via Suppression of TRAF6}

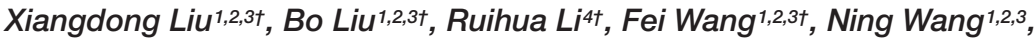

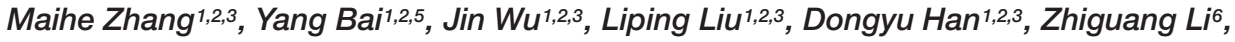 \\ Bin Feng ${ }^{7}$, Guangbiao Zhou ${ }^{8}$, Shujing Wang ${ }^{3,9}$, Li Zeng $^{1,2,3}$, Jian Miao ${ }^{5}$, Yiqun Yao ${ }^{10}$, \\ Bin Liang ${ }^{11}$, Lin Huang ${ }^{3,12 *}$, Qi Wang ${ }^{13 *}$ and Yingjie Wu ${ }^{1,2,3,14,15 *}$
}

\begin{abstract}
${ }^{1}$ Institute for Genome Engineered Animal Models of Human Diseases, Dalian Medical University, Dalian, China, ${ }^{2}$ National Center of Genetically Engineered Animal Models for International Research, Dalian Medical University, Dalian, China, ${ }^{3}$ Liaoning Provence Key Lab of Genome Engineered Animal Models, Dalian Medical University, Dalian, China, ${ }^{4}$ Department of Clinical Laboratory, Second Affiliated Hospital of Dalian Medical University, Liaoning, China, ${ }^{5}$ Division of Hepatobiliary and Pancreatic Surgery, Department of General Surgery, Second Affiliated Hospital of Dalian Medical University, Liaoning, China, ${ }^{6}$ Center of Genome and Personalized Medicine, Institute of Cancer Stem Cell, Cancer Center, Dalian Medical University, Dalian, China, ${ }^{7}$ Department of Biotechnology, Dalian Medical University, Dalian, China, ${ }^{8}$ Institute of Zoology, Chinese Academy of Sciences, Beijing, China, ${ }^{9}$ Department of Biochemistry and Molecular Biology, Institute of Glycobiology, Dalian Medical University, Dalian, China, ${ }^{10}$ Department of Thyroid and Breast Surgery, Affiliated Zhongshan Hospital of Dalian University, Dalian, China, ${ }^{11}$ School of Life Sciences, Yunnan University, Kunming, China, ${ }^{12}$ Department of Pathophysiology, College of Basic Medical Sciences, Dalian Medical University, Dalian, China, ${ }^{13}$ Department of Respiratory Medicine, Second Affiliated Hospital of Dalian Medical University, Liaoning, China, ${ }^{14}$ Department of Molecular Pathobiology, New York University College of Dentistry, New York, NY, United States, ${ }^{15}$ Division of Endocrinology, Diabetes and Bone Disease, Department of Medicine, Icahn School of Medicine at Mount Sinai, New York, NY, United States
\end{abstract}

Non-small cell lung cancer (NSCLC) is the most deadly cancer in the world due to its often delayed diagnosis. Identification of biomarkers with high sensitivity, specificity, and accessibility for early detection, such as circulating microRNAs, is therefore of utmost importance. In the present study, we identified a significantly higher expression of miR-146a-5p in the serum and tissue samples of NSCLC patients than that of the healthy controls. In parallel, miR-146a-5p was also highly expressed in three human NSCLC adenocarcinoma-cell lines (A549, H1299, and H1975) compared to the human bronchial epithelium cell line (HBE). By dual-luciferase reporter assay and manipulation of the expressions of miR-146a-5p and its target gene, tumor necrosis factor receptorassociated factor 6 (TRAF6), we showed that the functional effects of miR-146a-5p on NSCLC cell survival and migration were mediated by direct binding to and suppression of TRAF6. Overexpression of TRAF6 sufficiently reversed miR-146a-5p-induced cancer cell proliferation, migration, and apoptosis resistance. Our data implied that miR-146a5p/TRAF6/NF-кB-p65 axis could be a promising diagnostic marker and a therapeutic target for NSCLC.

Keywords: apoptosis, miR-146a-5p, migration, non-small cell lung cancer, TRAF6 


\section{INTRODUCTION}

Non-small cell lung cancer (NSCLC) is the most common form of lung cancer and accounts for approximately $80-85 \%$ of all lung cancers. Although several comprehensive treatments combining surgery, radiotherapy, and chemotherapy are used for NSCLC, it remains the most deadly cancer type in both genders (Zugazagoitia et al., 2014; Herbst et al., 2018). Thus, a more indepth understanding of cancer development is urgently needed for early diagnosis and targeted therapy of NSCLC.

MicroRNAs (miRNAs) are a group of small non-coding RNA molecules of less than 25 nucleotides and present across species from plants, animals to some viruses (Di Leva et al., 2014). miRNAs participate in the regulation of various physiological or pathological processes by direct binding to the $3^{\prime}$ untranslated regions ( $3^{\prime}$ UTR) of target genes, resulting in mRNA degradation or translational suppression (Polley et al., 2016; Perez-Sanchez et al., 2018). Although the miRNA-guided post-transcriptional control was mainly revealed in the immune system, the evidence is piling up to link it with a great number of other disorders, such as systemic lupus erythematosus (SLE) and common cancers in colon, stomach, ovary, cervical, prostate, and lung (Paik et al., 2011; Hung et al., 2013; Di Leva et al., 2014; Zheng et al., 2015; Polley et al., 2016; Jiang et al., 2017; Yin et al., 2017; Hu et al., 2018; Li et al., 2019). Similar to protein-coding genes, miRNAs can function as either oncogenes or tumor suppressors to be potential prognostic biomarkers (Thai et al., 2010; Jalava et al., 2012; Wang et al., 2016).

As one of the miRNAs implicated in common cancers, miRNA-146a is associated with cancer progression, positively or negatively. It was demonstrated to be expressed significantly higher in the plasma samples of breast cancer patients than those of healthy controls (Kumar et al., 2013; Jiang et al., 2017). There are reports showing the association between increased tissue miR146a expression and tumor size or tumor recurrence in human oesophageal squamous cell carcinoma (Zhou et al., 2017), and its anti-apoptotic activity in gastric cancer (Xiao et al., 2012). However, a negative impact of miR-146a on cancer development was also reported in prostate cancer (Meng et al., 2020), head and neck squamous cell carcinoma (Lerner et al., 2016), cervical cancer (Hu et al., 2018), and NK/T cell lymphoma (Paik et al., 2011). Not only does the role of miRNA-146a seem to be cancer type-dependent, but also opposing conclusions were reached in studies of NSCLC. One study reported that miR-146a-5p was downregulated in NSCLC tissues (Wu et al., 2014), whereas another study indicated that miR-146a-5p was up-regulated in the serum of NSCLC patients (Wang et al., 2015). Sample size, cancer subtypes and stage, patient background, measurement approaches, and even compensation of miRNA-146b (Paterson and Kriegel, 2017), could be the factors compounding the confusion in publications and await thorough clarification. miR146a biogenesis and its binding ability to its target genes are also subject to the control of single nucleotide polymorphisms (SNPs), the most common form of DNA variation in the human genome that may effectually contribute to cancer susceptibility (Duan et al., 2007). SNP variance in miR-146a precursor rs2910164 was related to anti-TNF-a treatment in rheumatoid arthritis
(RA) patients (Bogunia-Kubik et al., 2016) and also affected phenotypes in breast cancer (BC; Meshkat et al., 2016) and the progression of cervical cancer (Hu et al., 2018). In a female nonsmoking lung cancer cohort from Northeast China, a strong association between miR-146a rs2910164 CG or GG genotype and a lower lung cancer risk than the wild-type homozygous CC genotype was found, which might be due to reduced binding of SNP variants to its target gene tumor necrosis factor receptorassociated factor 6 (TRAF6; Yin et al., 2017).

As a signal transducer in the miR-146a-5p/TRAF6/NF- $\kappa$ B axis that fine-tunes immune homeostasis, TRAF6 is a downstream effector of miR-146a-5p, with pleiotropic roles in carcinogenesis, cancer invasion and metastasis (Meng et al., 2012; Zhang X.L. et al., 2014). Via down-regulation of TRAF6, miR-146a-5p suppressed hepatocellular carcinoma (HCC) cell proliferation and invasion in vitro and inhibited tumor formation in vivo and in vitro ( $\mathrm{Zu}$ et al., 2016). miR-146a was not only overexpressed in cervical cancer and promoted cancer cell proliferation by targeting TRAF6 through NF- $\mathrm{KB}$ signaling (Li et al., 2019), but also in oral carcinoma by targeting IRAK1, TRAF6, and NUMB (Hung et al., 2013; Min et al., 2017). miR-146a-5p/TRAF6/NFкB-p65 axis regulated cell growth and gemcitabine (GEM) chemotherapy sensitivity in pancreatic ductal adenocarcinoma (PDAC; Meng et al., 2020). However, where the expression of TRAF6 in NSCLC is concerned, discrepant conclusions are drawn. In one cohort, TRAF6 expression was higher in the tumor than surrounding tissues in 59.6\% of the patients and was inversely related to chemo-sensitivity, but unrelated to cancer stages or overall survival (Liu et al., 2012). In another similarsized study, a positive correlation was detected between TRAF6 positivity and cancer stage of both NSCLC and SCLC (Zhang X.L. et al., 2014). Over-expression of TRAF6 gene aligned with the amplification of chromosome 11 at band p13 was proposed to constitute the MAPK pathway activation in human lung cancer development (Starczynowski et al., 2011). Whether the murky picture of TRAF6 in NSCLC coincides or has a mechanistic relationship with miR-146a-5p regulation was not analyzed in these reports and is to be addressed here.

In this study, we determined the status of miR-146a-5p in the serum and tissue samples from NSCLC patients and in the NSCLC cell lines, its role in cancer cell proliferation and migration. We identified TRAF6 as the important target gene of miR-146a-5p to promote NSCLC cancer survival.

\section{MATERIALS AND METHODS}

\section{Tissue and Serum Samples}

Serums from a total of 36 NSCLC cancer patients and 11 healthy controls and 16 NSCLC cancer tissues with corresponding adjacent paracancerous tissue specimens were obtained from the Second Affiliated Hospital of Dalian Medical University (Dalian, China). The healthy control samples were collected from individuals seeking routine physical health examination in the hospital and having negative results in lung cancer screening. All subjects were thoroughly informed in writing about the research procedure and have given consent to participate. The 
study protocol was approved by the Ethics Committee of Second Affiliated Hospital of Dalian Medical University. During the surgical treatment, all tissues were immediately stored at $-80^{\circ} \mathrm{C}$ until the RNA and protein were later extracted for experiments. In addition, the related information on the clinical samples is shown in Supplementary Figure S1.

\section{Cell Culture}

The human bronchial epithelium cell line (HBE) and NSCLC cell lines A549, H1299, and H1975, were obtained from American Type Culture Collection (ATCC, Manassas, VA, United States). All cell lines were cultured in RPMI-1640 medium (Thermo Fisher Scientific, Waltham, MA, United States) containing 10\% fetal bovine serum (FBS; Gemini Bio Products, West Sacramento, CA, United States), $100 \mathrm{IU} / \mathrm{ml}$ penicillin and $100 \mu \mathrm{g} / \mathrm{ml}$ streptomycin (Solarbio ${ }^{\circledR}$ Life Sciences, Beijing, China), at $37^{\circ} \mathrm{C}$ with $5 \% \mathrm{CO}_{2}$ in a humidified incubator (NuAire, Plymouth, MN, United States).

\section{Cell Transfection}

Cells were plated in 24-well plates or $10 \mathrm{~cm}$ dishes. When reached 70-80\% confluence, cells were transfected with $100 \mathrm{nM}$ miR146a-5p mimics, miR-146a-5p inhibitor, siTRAF6 and 500 ng plasmids (GenePharma, Suzhou, China), using Lipofectamine 3000 reagent (Invitrogen, Carlsbad, CA, United States) following the manufacturer's instruction. All oligonucleotide sequences are listed in Supplementary Table S1.

\section{RNA Extraction and qRT-PCR}

Total RNA was extracted from frozen tissues or cultured cells using RNAiso Plus Reagent (Takara, Dalian, China), according to the manufacturer's protocols. cDNA was synthesized from mRNA using the TransScript One-Step gDNA Removal and cDNA Synthesis SuperMix (Transgene, Beijing, China), and miRNA reverse transcription was performed using OneStep miRNA cDNA Synthesis Kit (Transgene), following the manufacturer's instruction. The levels of mRNA or miRNA were quantified by qRT-PCR, using the TransStart Tip Green qPCR SuperMix (Transgene). The endogenous controls for mRNA and miRNA were GAPDH and U6, respectively. The data were calculated using the $2^{-\Delta \Delta C T}$ method. Primer sequences are listed in Supplementary Table S2.

\section{Cell Proliferation Assay}

Cells were plated in 96-well plates (Corning, New York, United States) at a density of $3 \times 10^{3} /$ well and transfected with miRNA mimics/inhibitors or siRNA at about $40 \%$ confluency. Cell viability was assessed at $0,24,48$, and $72 \mathrm{~h}$ after transfection by adding $10 \mu \mathrm{l}$ of Cell Counting Kit-8 (CCK-8; Transgene) reagent and $90 \mu \mathrm{l}$ non-serum RPMI-1640 into each well and incubation for $30 \mathrm{~min}$. The absorbance at $450 \mathrm{~nm}$ was measured using an EMax Plus Microplate Reader (Molecular Devices, San Jose, CA, United States).

\section{Immunofluorescence Assay}

Cells were plated in 24-well plates and grown to about $40 \%$ confluence when transfected with miRNA mimics/inhibitors or siRNA for $24 \mathrm{~h}$. Cells were then fixed with $10 \%$ neutral buffered formalin (Coolaber, Beijing, China) for $30 \mathrm{~min}$ at room temperature. The fixed cells were incubated with a primary rabbit antibody against ki-67 (Proteintech, Beijing, China) overnight at $4^{\circ} \mathrm{C}$. After incubation with Dylight 594 conjugated goat anti-rabbit IgG secondary antibody (Abbkine, Redlands, CA, United States) for $2 \mathrm{~h}$ at room temperature, the 4, 6-Diamidino-2-phenylindole (DAPI; Sigma Corporation of America, Ronkonkoma, NY, United States) at 1:2000 was added for $30 \mathrm{~min}$. The cells were visualized using a fluorescence microscope (Nikon ECLIPSE Ni-E, Tokyo, Japan).

\section{Transwell Migration Assay}

Twenty-four-well plates and $8-\mu \mathrm{m}$ Transwell cell culture chambers (Promega Corporation, Madison, WI, United States) were utilized for transwell assay. Briefly, $1 \times 10^{5}$ cells in $200 \mu \mathrm{l}$ serum-free medium were placed into the upper chamber. RPMI-1640 medium containing 10\% FBS (Gemini Bio Products) was added into the lower chamber as a chemo-attractant. Cells that did not migrate after $24 \mathrm{~h}$ in the upper part of the filters were removed with a cotton swab. The membranes were next fixed by $10 \%$ neutral buffered formalin (Coolaber) for $30 \mathrm{~min}$ and stained with $0.5 \%$ crystal violet (Beyotime Biotechnology, Shanghai, China) for $10 \mathrm{~min}$. The membranes were washed thoroughly with $1 \times$ PBS and dissolved in $500 \mu 133 \%$ acetic acid. The migrated cells were detected at $200 \times$ or $100 \times$ magnification in at least six different fields of each filter and the absorbance was measured at $570 \mathrm{~nm}$.

\section{Wound Healing Assay}

Cells were placed into six-well plates at a density of $5 \times 10^{4} /$ well. When the cells were $90 \%$ confluent, a straight wound line was scraped with a $10 \mu \mathrm{l}$ pipette tip in each well. Pictures were snapped before and after the subsequent $24 \mathrm{~h}$ incubation, using a phase-contrast microscope (Nikon ECLIPSE TS100). The cell migration rate in the scratch areas was quantified by ImageJ software.

\section{Apoptosis Analysis}

After transfection with miR-146a-5p mimics, miR-146a-5p inhibitor, siTRAF6 and TRAF6-overexpression plasmids or their negative controls for $48 \mathrm{~h}$, cells were resuspended in $200 \mu \mathrm{l}$ Binding Buffer (Solarbio ${ }^{\circledR}$ Life Sciences, Beijing, China) at $5 \times 10^{5} / \mathrm{ml}$. Cell suspension was incubated in $10 \mu \mathrm{l}$ Annexin V-FITC (Solarbio ${ }^{\circledR}$ Life Sciences) and $10 \mu \mathrm{l}$ propidium iodide (PI; Solarbio ${ }^{\circledR}$ Life Sciences) for $15 \mathrm{~min}$ in the dark. The reaction was terminated by adding $300 \mu \mathrm{l}$ Binding Buffer and analyzed by flow cytometry (BD FACS Canto II, Franklin Lakes, NJ, United States). Cells stained positive for FITC-Annexin were considered apoptotic. TRAF6 ORF was cloned into pEX-3 vector (GenePharma, Shanghai) at XhoI/EcoRI sites under the control of CMV promoter. The complete plasmid map and sequence are available upon request.

\section{Dual-Luciferase Reporter Assay}

To demonstrate the direct interaction between miR-146a-5p and TRAF6, the 3' UTR sequence of TRAF6 mRNA containing the 
putative miR-146a-5p binding sites was synthesized by Sangon Biotech (Shanghai, China), and sub-cloned to downstream of the firefly reporter gene in the pmirGLO vector (Promega, Madison, WI, United States), which was named pmirGLO-TRAF6-WT. Mutant TRAF6 3' UTR, containing a mutated binding site for miR-146a-5p, was cloned into pmiRGLO and named pmiRGLOTRAF6-MUT. The oligonucleotide sequences are listed in Supplementary Table S1. For the dual luciferase reporter assay, cells were cultured in a 96-well plate and co-transfected with $100 \mathrm{nM}$ miR-146a-5p mimics and $200 \mathrm{ng}$ pmirGLO-TRAF6WT reporter plasmid or pmiRGLO-TRAF6-MUT vector using Lipofectamine 3000 (Invitrogen). The luciferase activity was measured $48 \mathrm{~h}$ after transfection, using the Dual-Glo Luciferase Assay System (Promega) and assayed with Multimode Plate Reader (Coring, NY, United States). The relative transcriptional activities were expressed as the fold change above the vehicle control in luciferase activity after normalization to renilla luciferase activity.

\section{Western Blot}

At $24 \mathrm{~h}$ post-transfection, total proteins were extracted from the cells using RIPA buffer (Solarbio ${ }^{\circledR}$ Life Sciences), and quantified with a Protein BCA Assay Kit (Beyotime Biotechnology). The protein lysates were separated by sodium dodecyl sulfatepolyacrylamide gel electrophoresis (SDS-PAGE) and transferred to polyvinylidene difluoride (PVDF) membrane (PALL, Ann Arbor, MI, United States). After blocking in 5\% skimmed milk (BD, San Jose, CA, United States) at room temperature for $2 \mathrm{~h}$, the membranes were blotted with specific primary antibodies overnight at $4^{\circ} \mathrm{C}$. The membranes were probed with secondary antibodies conjugated to horseradish peroxidase (HRP; Abbkine) after washing with $1 \times$ TBST. The protein bands were detected using ECL solution (Abbkine) and imaged with BIO-RAD system (Bio-Rad Laboratories, Stuttgart, Germany). Primary antibody information is listed in Supplementary Table S3.

\section{Statistical Analysis}

All numerical data were presented as mean \pm SD. Statistical analysis was carried out with Student's $t$-test in GraphPad Prism6. Data were considered to be significantly or very significantly different when $p<0.05$ (marked with ${ }^{*}$ ) or $p<0.01$ (marked with $\left.{ }^{* *}\right)$, respectively.

\section{RESULTS}

\section{miR-146a-5p Is Overexpressed in NSCLC Serum, Tissues and NSCLC Cell Lines}

To determine the miR-146a-5p expression level in the serum of NSCLC patients and the healthy controls, miRNA Solexa sequencing (Figure 1A) and qRT-PCR (Figure 1B) were conducted. Both results showed that the expression of miR146a-5p was significantly higher in NSCLC serum than in the normal serum samples. Measured by qRT-PCR, miR-146a-5p gene expression in NSCLC cancer tissues had a five-fold increase compared to the corresponding paracancerous tissues
(Figure 1C), and was significantly overexpressed in three NSCLC cell lines (A549, H1299, and H1975) when compared with the human bronchial epithelium cell line (HBE; Figure 1D). miR-146a-5p was overexpressed in lung squamous cell carcinoma (LUSC) and lung adenocarcinoma (LUAD) from TCGA database (Figure 1E). These results suggested a potential involvement of miR-146a-5p in the initiation and tumorigenesis of NSCLC. A549 and H1299 cells were chosen for subsequent overexpression and down-regulation experiments, as they had the lowest and highest miR-146-5p expression among the tested NSCLC cell lines, respectively.

\section{Overexpression of miR-146a-5p Promotes the Proliferation and Migration of A549 Cells}

To evaluate the biological effects of miR-146a-5p on NSCLC cell lines, miR-146a-5p mimic (miR-146a-5p) or its corresponding negative control (miR-NC) was transfected into A549 cells. The transfection efficiency was confirmed by significant up-regulation of miR-146a-5p gene expression in A549 cells after transfection (Figure 2A). In the meantime, cell proliferation of A549 cells, as determined by CCK- 8 assay, was enhanced by miR-146a-5p overexpression (Figure 2B). Since the cells sustained a stable higher rate of proliferation within 3 days after transfection, the time point to harvest the cells for other cellular assays was set at $24 \mathrm{~h}$ post-transfection. The indication of active cell proliferation promoted by miR-146a-5p was reinforced by strong immunofluorescence staining of Ki-67, a validated index of lung cancer malignancy (Warth et al., 2014; Figures 2C,D). The transwell assay result (Figures 2E,F) and wound healing assay (Figures 2G,H) showed that the cells transfected with miR-146a$5 \mathrm{p}$ mimic had a higher migratory capacity.

\section{Suppression of miR-146a-5p Inhibits the Proliferation and Migration of H1299 Cells}

To further study the effects of miR-146a-5p on NSCLC cell growth from another angle, miR-146a-5p inhibitor or its corresponding control (NC inhibitor) was transfected into H1299 cells. In contrast to miR-146a-5p overexpression, suppression of miR-146a-5p in H1299 cells by miR-146a-5p inhibitor (Figure 3A) led to reduced cell proliferation, shown by both CCK-8 assay (Figure 3B) and Ki-67 staining (Figures 3C,D). Also, the transwell assay (Figures 3E,F) and wound healing assay (Figures 3G,H) concomitantly displayed a reduced migratory capacity of the cells transfected with miR-146a-5p inhibitor. Taken together, loss- and gain-of-function of miR-146a-5p resulted in an overt reversal of the cancer cell behavior.

\section{miR-146a-5p Inhibits Apoptosis of NSCLC Cells}

As a strategy of advantageous survival, evading apoptosis is one of the major hallmarks of cancer coined by Hanahan and Weinberg (2000). The influence of miR-146a-5p on apoptosis of NSCLC was examined in A549 and H1299 cells transfected with miR-146a-5p mimic (miR-146a-5p) or miR-146a-5p inhibitor, 
A

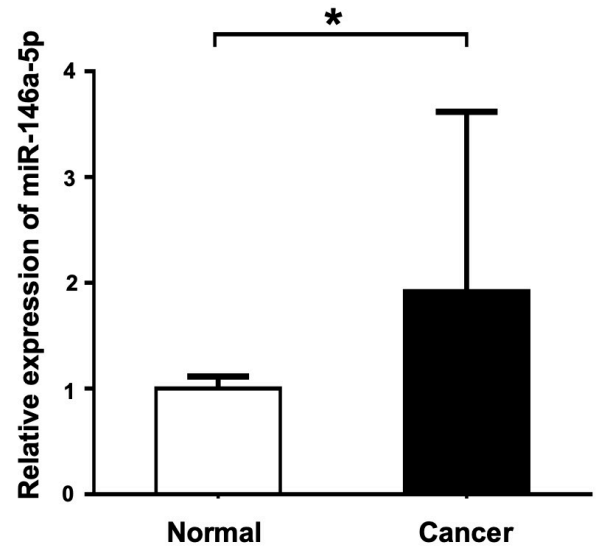

C

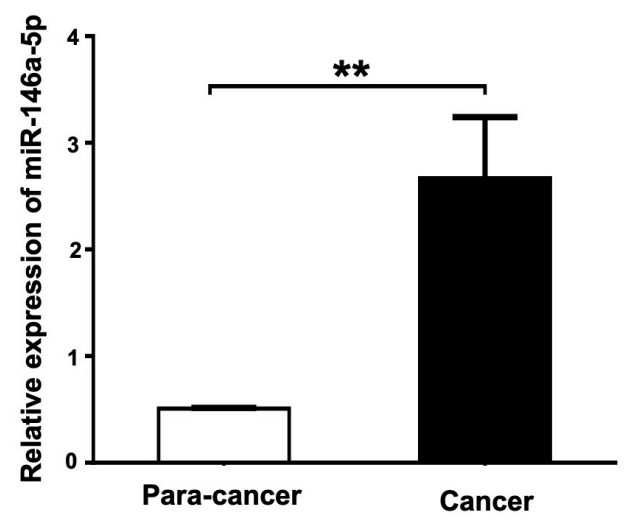

B

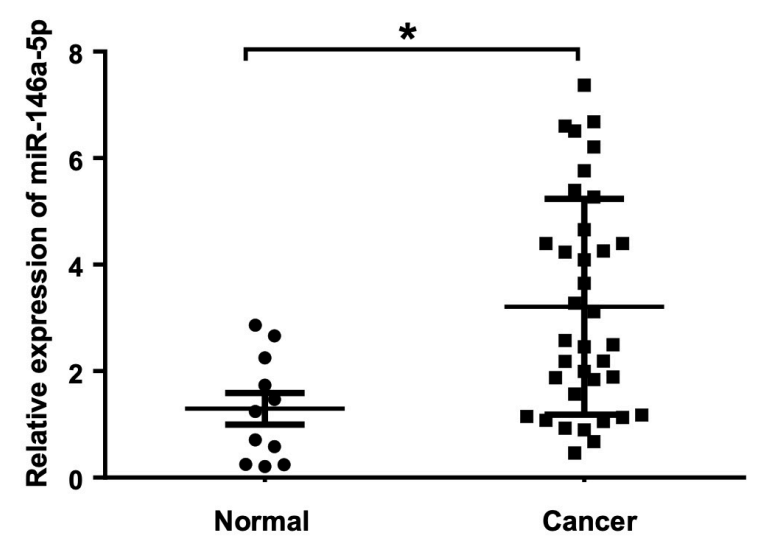

D

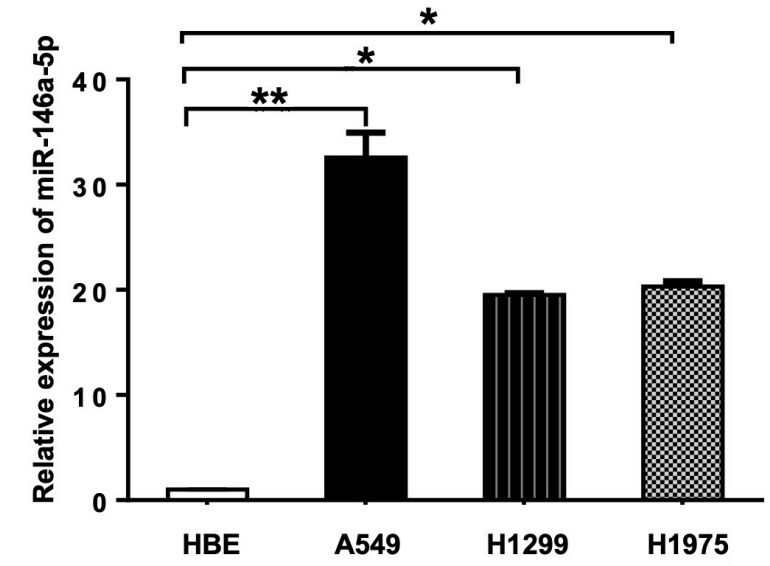

$\mathbf{E}$

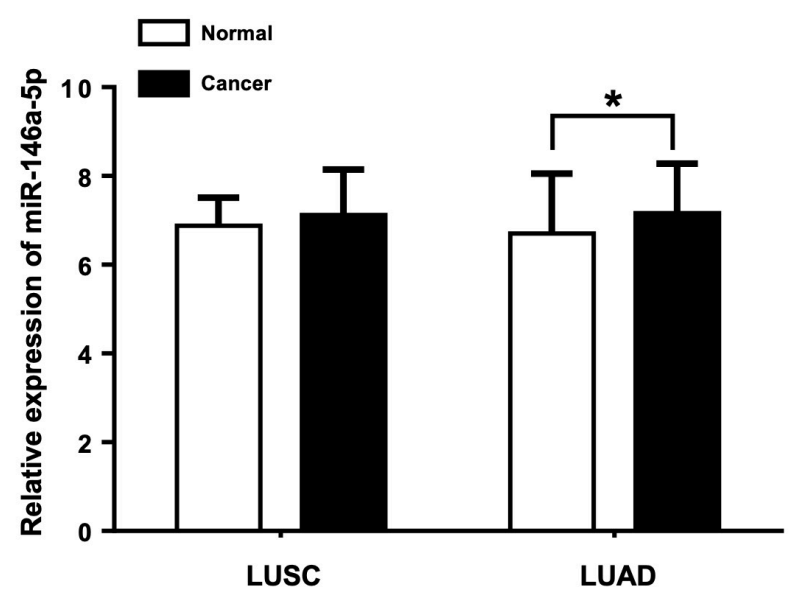

FIGURE 1 | miR-146a-5p expression is up-regulated in NSCLC tissues, serum samples and cell lines. (A) Expression of miR-146a-5p in NSCLC patient and normal serum samples measured by miRNA Solexa sequencing. (B) qRT-PCR validation of the result from (A). (C) Expression of miR-146a-5p in NSCLC cancer and corresponding para-cancer tissues determined by qRT-PCR. (D) Expression of miR-146a-5p in three NSCLC cell lines (A549, H1299 and H1975) and human bronchial epithelium cell line (HBE) determined by qRT-PCR. (E) Expression of miR-146a-5p from TCGA. LUSC: lung squamous cell carcinoma, LUAD: lung adenocarcinoma. 
A

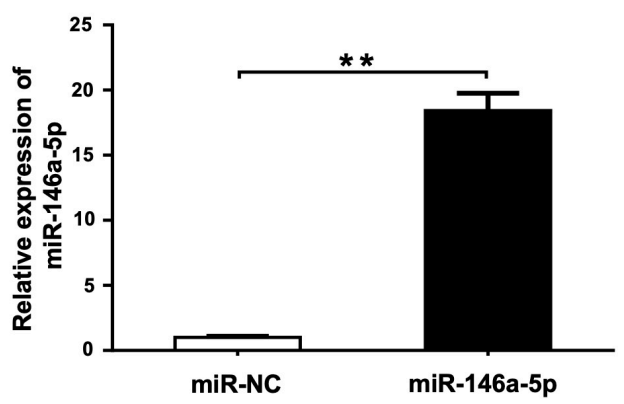

C

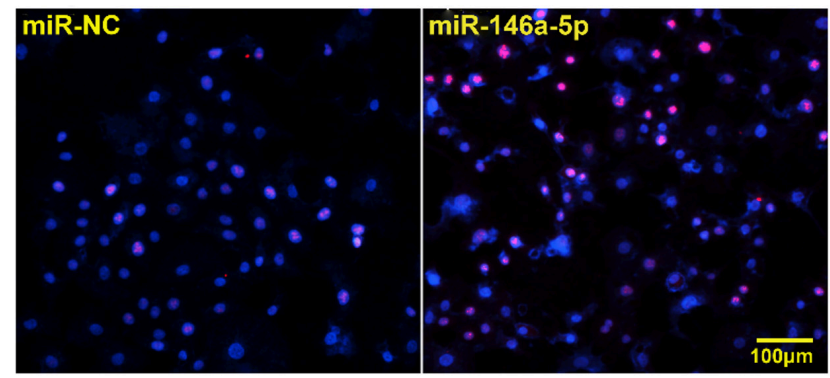

E

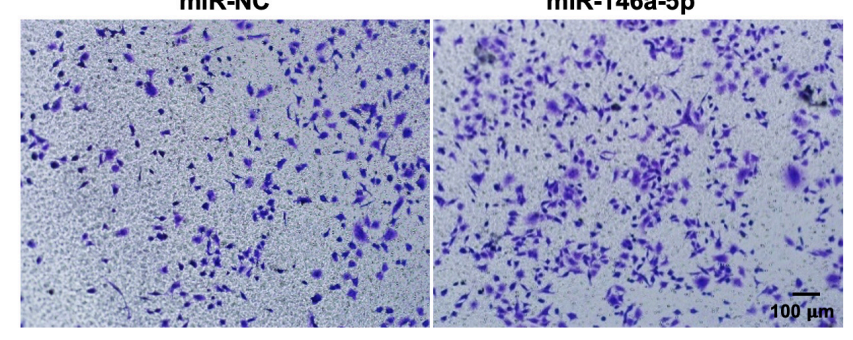

G

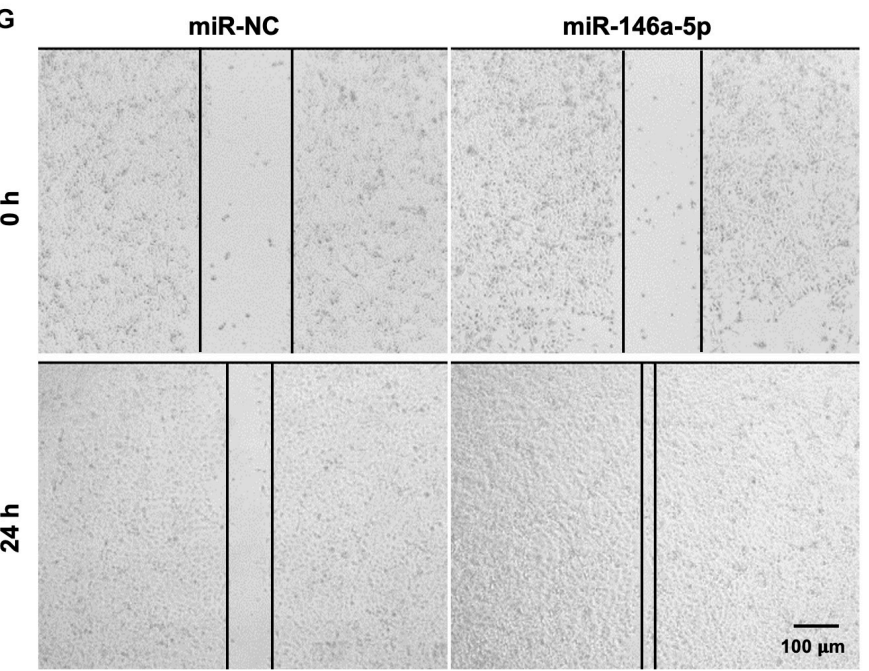

B

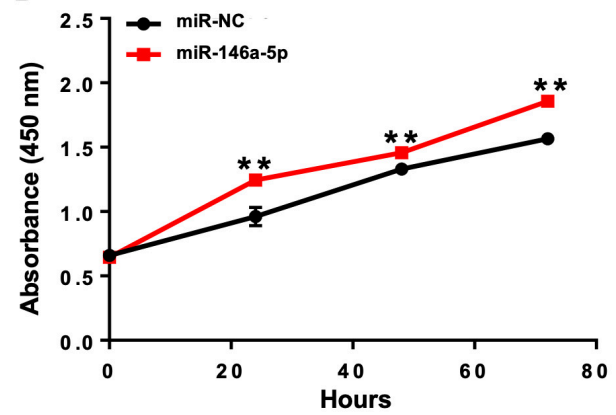

D

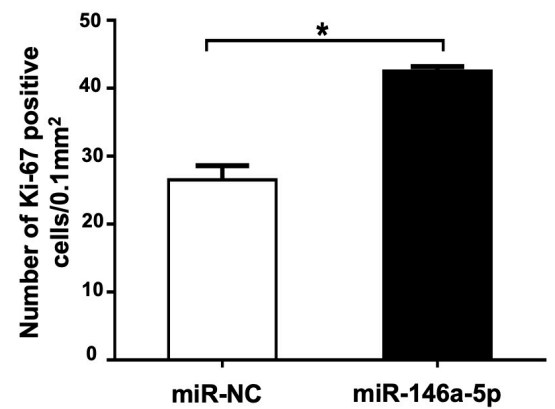

$\mathbf{F}$

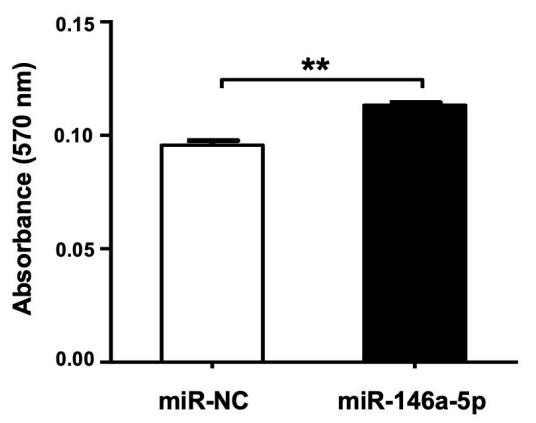

H

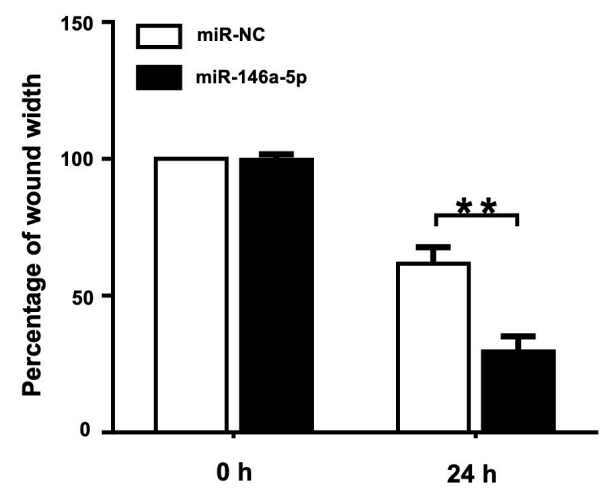

FIGURE 2 | miR-146a-5p overexpression promotes cell proliferation and migration in NSCLC cells. Cell proliferation and migration measurement of A549 cells at $24 \mathrm{~h}$ post transfection of miR-146a-5p mimic or miR-NC. (A) The mRNA expression of miR-146a-5p measured by qRT-PCR. (B) Cell proliferation examined by CCK-8 assay. (C) Cell proliferation measured by Ki-67 staining. (D) Quantification of the Ki-67 staining assay result of (C). (E) Migration ability measured by transwell assay. (F) Quantification of the transwell assay result of (E). (G) Migration ability detected by wound-healing assay. (H) Quantification of the wound-healing assay result of (G). 
A

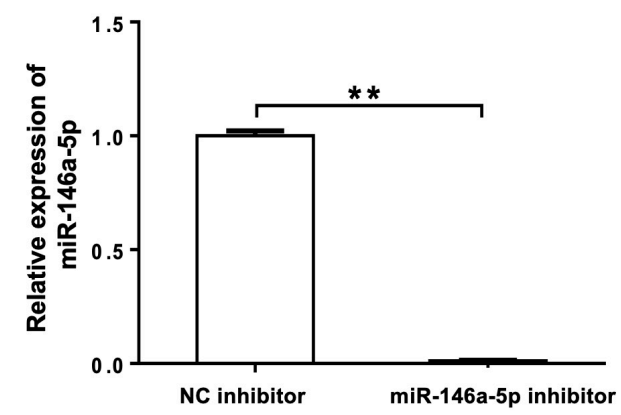

C

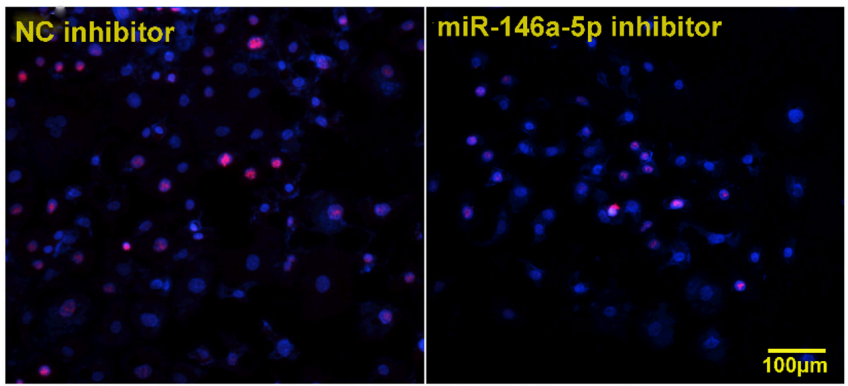

E

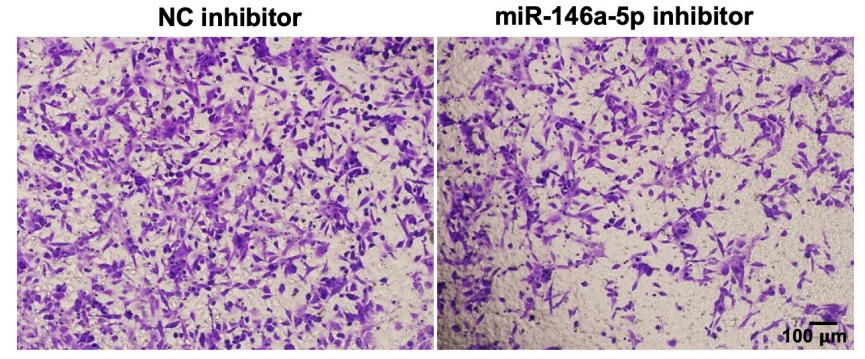

G

$\mathbf{G}$

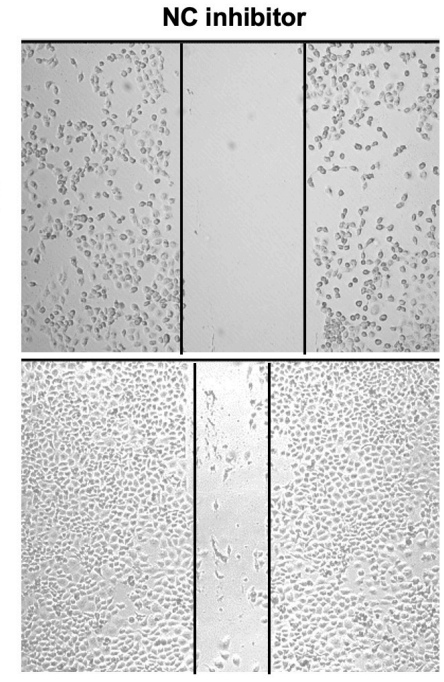

miR-146a-5p inhibitor

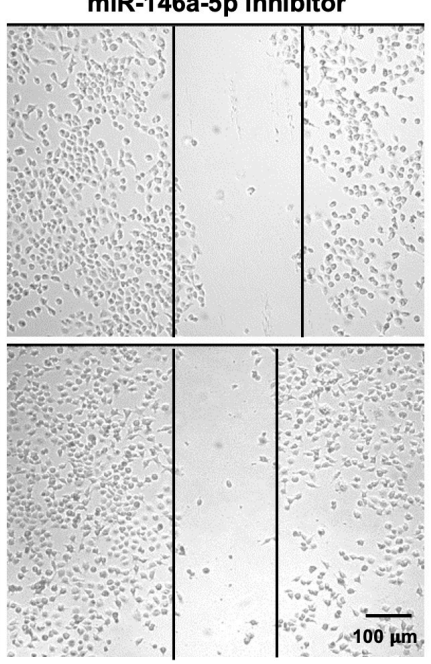

B

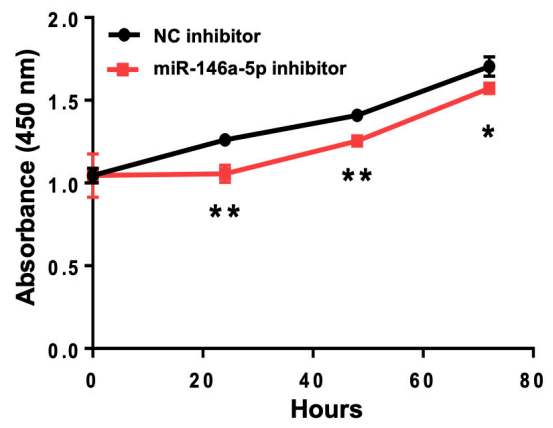

D

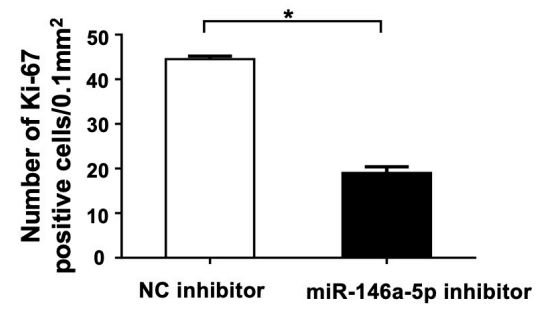

$\mathbf{F}$

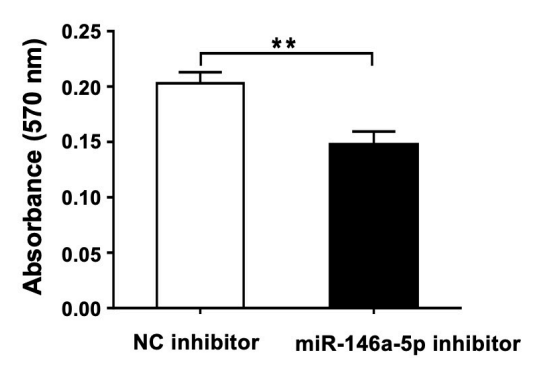

H

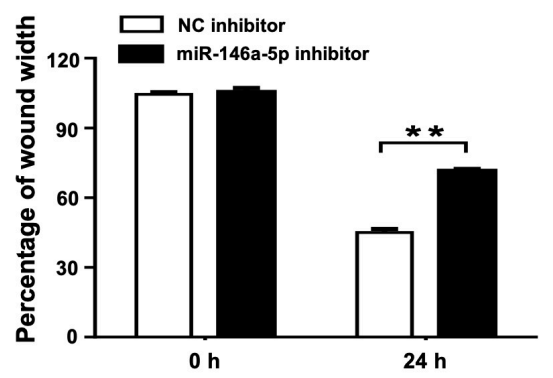

FIGURE 3 | miR-146a-5p inhibitor suppresses cell proliferation and migration in NSCLC cells. Cell proliferation and migration measurement of H1299 cells at $24 \mathrm{~h}$ post transfection of miR-146a-5p inhibitor or NC inhibitor. (A) The mRNA expression of miR-146a-5p measured by qRT-PCR. (B) Cell proliferation examined by CCK-8 assay. (C) Cell proliferation measured by Ki-67 staining. (D) Quantification of the Ki-67 staining assay result of (C). (E) Migration ability measured by transwell assay. (F) Quantification of the transwell assay result of (E). (G) Migration ability detected by wound-healing assay. (H) Quantification of the wound-healing assay result of (G). 
respectively. Flow cytometric analysis demonstrated that the upregulation of miR-146a-5p resulted in a lower percentage of apoptotic A549 cells (Figures 4A,C) and down-regulation of miR-146a-5p resulted in a higher percentage of apoptosis in H1299 cells (Figures 4B,D). Western blot was used to determine the apoptosis-related genes $\mathrm{Bcl}-2$ and $\mathrm{Bax}$. The $\mathrm{Bax} / \mathrm{Bcl}-2$ ratio, which predisposes cell susceptibility to apoptosis (Jaeschke et al., 2018), was decreased by miR-146a-5p overexpression and increased by miR-146a-5p inhibition (Figures 4E,G). Meanwhile, Western blot data showed that miR-146a-5p decreased the phosphorylation level of NF-кB-p65 (pS536; Figures 4E,F) that is required for $\mathrm{NF}-\kappa \mathrm{B}$ nuclear translocation and its transcriptional activity, implying miR-146a-5p to be a negative regulator of NF- $\kappa \mathrm{B}$ signaling pathway. At the same time, miR$146 a-5 p$ decreased the level of cleaved caspase 3 and cleaved PARP (Figures 4E,H-I), indicating that miR-146a-5p conferred resistance to apoptosis in NSCLC cells.

\section{miR-146a-5p Directly Targets TRAF6 in NSCLC Cells}

Using three different databases including miRDB, TargetScan, and PicTar, we identified TRAF6 as one of the targets in the intersection between the groups of predicted targets of miR146a-5p (Figure 5A). In comparison to the negative control, the mRNA expression and protein level of TRAF6 were decreased in miR-146a-5p mimic (miR-146a-5p)-transfected A549 cells (Figures 5B,C). Consistently, miR-146a-5p inhibitor treatment led to a significant up-regulation of TRAF6 at mRNA and protein levels in H1299 cells (Figures 5B,C). The correlated expression of TRAF6 in NSCLC was next investigated by mining the clinical data from TCGA ${ }^{1}$. A lower expression of TRAF6 was revealed in both lung squamous cell carcinoma (LUSC) and lung adenocarcinoma (LUAD) than in adjacent normal lung tissues (Figure 5D and Supplementary Figures S2A-C). The TRAF6 protein levels were decreased in human NSCLC cancer tissues compared with the paired paracancerous tissues (Figures 5E,F), and were lower in NSCLC cell lines than in the human bronchial epithelium cell line (HBE; Figures 5G,H). According to the miRNA databases, a putative miR-146a-5p binding site located in the $3^{\prime}$ UTR of TRAF6 mRNA was identified. To test whether the TRAF6 gene transcription is directly hindered by miR-146a-5p, the $3^{\prime}$ UTR of TRAF6 sequence that includes the binding site of miR-146a-5p was cloned into the pmiR-GLO plasmids (a dual-luciferase reporter), named pmiRGLO-TRAF6-WT. As control of binding specificity, a pmiRGLO-TRAF6-MUT was constructed by replacement of the binding site with a $3^{\prime}$ UTR mutation (Figure 5I). The pmiRGLO-TRAF6-WT or pmiRGLO-TRAF6-MUT were cotransfected with either miR-146a-5p mimic (miR-146a-5p) or miR-NC into A549 cells. A remarkable reduction of the relative luciferase activity was only observed in cells co-transfected with miR-146a-5p and pmiRGLO-TRAF6-WT, not in cells cotransfected with other combinations (Figure 5J), suggesting that the $3^{\prime}$ UTR sequence of TRAF6 is indeed a direct target gene of miR-146-5p.

${ }^{1}$ https://www.cancer.gov

\section{Knockdown of TRAF6 Promotes the Proliferation and Migration, and Inhibits Apoptosis of NSCLC Cells}

To demonstrate the importance of TRAF6 in mediating the pro-survival effects of miR-146a-5p in NSCLC cells, two TRAF6 siRNAs targeting different regions, siTRAF6-1 and -2 , and the scrambled control (siNC) were transfected into A549 cells. qRT-PCR results showed that TRAF6 gene was significantly silenced by 75.66 and $69.86 \%$ by siTRAF6-1 and -2 , respectively (Figure 6A). siTRAF6-1 was used for further experiments because of its higher silencing capacity. The efficiency of TRAF6 silencing by siTRAF6-1 was also confirmed by decreased TRAF6 protein level as shown in Western blot (Figures 6B,C). In agreement with our hypothesis, the above-observed effects of miR-146a-5p overexpression in A549 cells were recapitulated by TRAF6 knockdown, encompassing enhanced cell proliferation (Figures 6D,E), higher migratory capacity (Figures 6F-I) and a lower percentage of apoptotic cells (Figures 6J,K).

\section{Overexpression of TRAF6 Inhibits the Proliferation and Migration, and Promotes Apoptosis of NSCLC Cells}

To investigate the effects of TRAF6 in NSCLC cells, the TRAF6 overexpression (TRAF6 OE) and control (empty vector) were transfected into H1299 cells. TRAF6 was significantly overexpressed at both mRNA (Figure 7A) and protein level (Figures 7B,C). In cells with TRAF6 overexpression, inhibited cell proliferation was confirmed by CCK- 8 assay (Figure 7D) and ki-67 staining (Figures 7E,F), while impeded cell migration was detected by transwell (Figures $\mathbf{7 G}, \mathbf{H}$ ) and wound healing assay (Figures 7I,J). However, the percentage of apoptosis in TRAF6overexpressing H1299 cells was higher than in the control, albeit to a lesser extent (Figures $7 \mathbf{K}, \mathbf{L}$ ).

\section{Overexpression of TRAF6 Reverses the Malignant Behavior of NSCLC Cells Potentiated by miR-146a-5p Overexpression}

A rescue experiment was designed to determine whether restoration of TRAF6 expression in miR-146a-5p overexpression cells is enough to reverse the oncogenic effects of miR-146a-5p. H1299 cells were parallelly transfected with miR-NC, miR146a-5p mimic (miR-146a-5p) or co-transfected with miR-146a-5p mimic plus TRAF6 overexpression plasmid (miR-146a-5p+TRAF6), respectively. As expected, the examined cancer-specific characteristics of the cells caused by miR146-5p overexpression were all reversed by TRAF6 plasmid co-transfection. The reduced protein level of TRAF6 by miR-146-5p was fully recovered by the ectopic expression of TRAF6 (Figures 8A,B). The ability of miR-146a-5p to enhance proliferation (Figure $8 \mathrm{C}$ ), promote migration (Figures 8D-G) and inhibit apoptosis (Figures $\mathbf{8 H}, \mathbf{I}$ ) in H1299 cells was fully reversed by forced expression of TRAF6. 
A

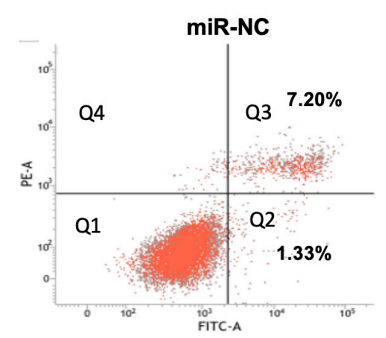

B

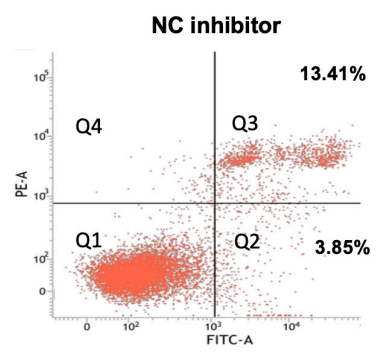

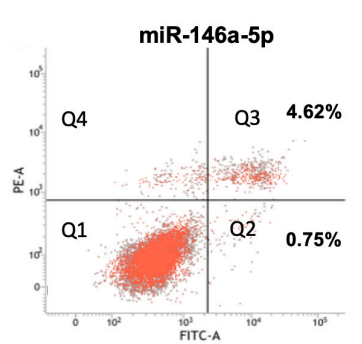

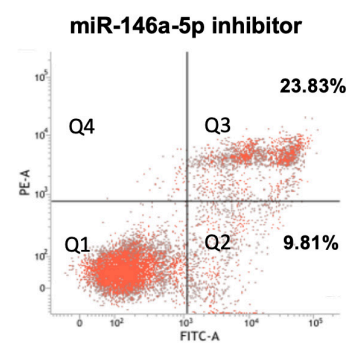

E

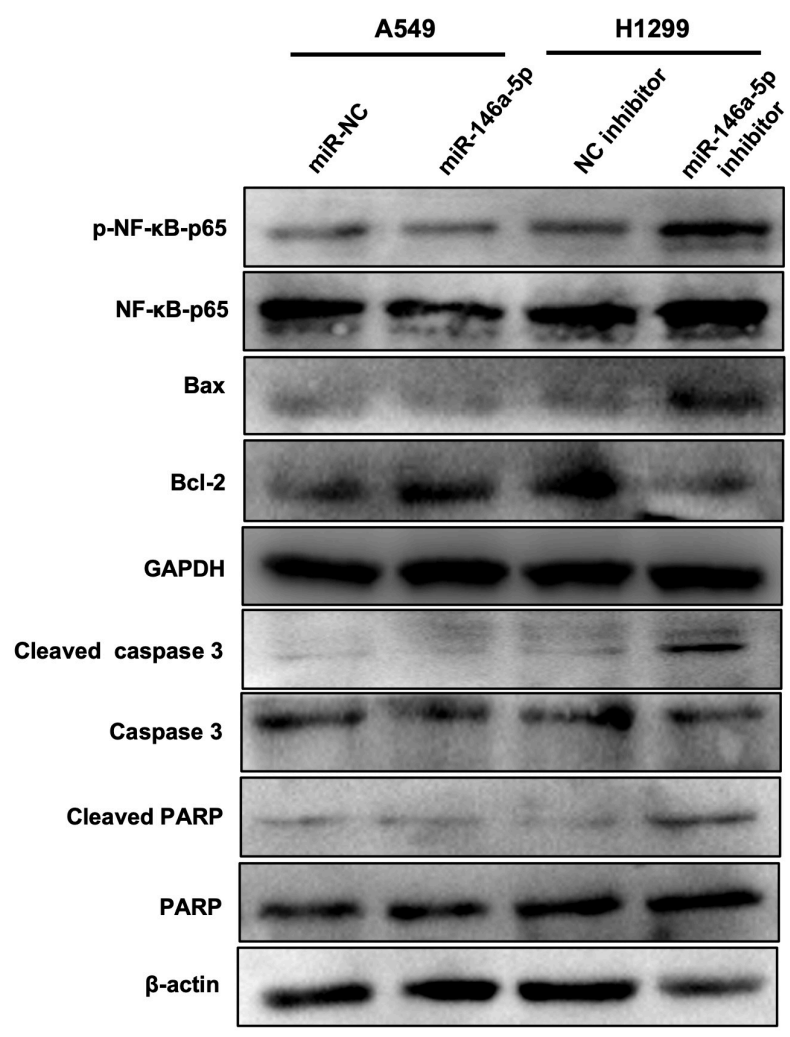

C
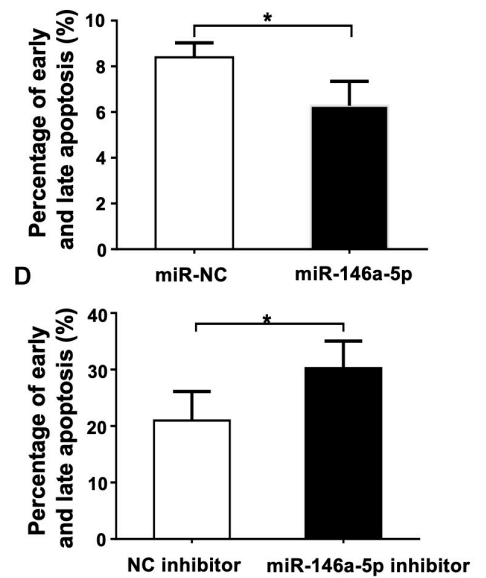

$\mathbf{F}$

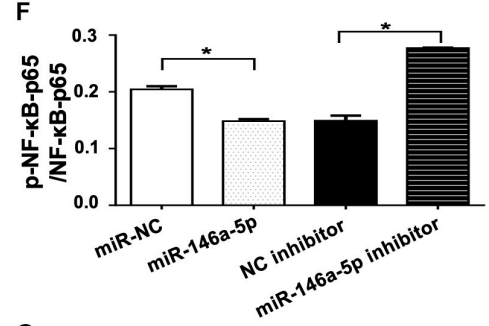

G
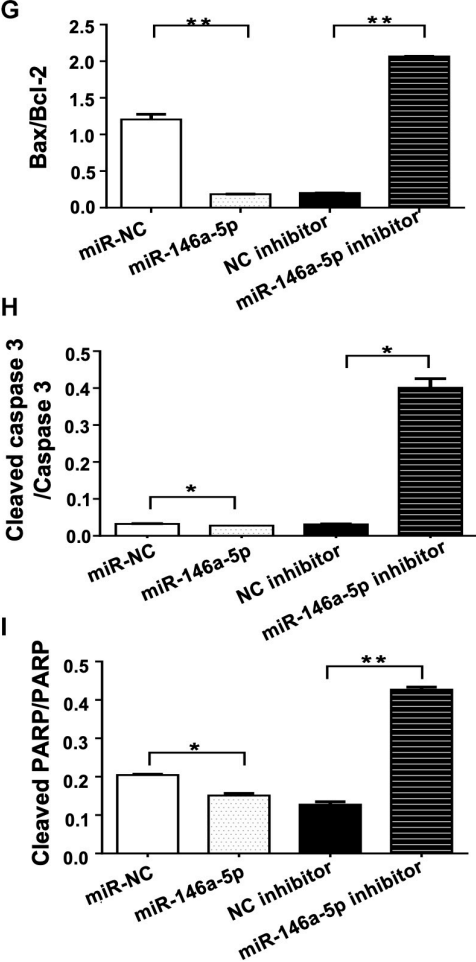

FIGURE 4 | miR-146a-5p inhibits apoptosis in NSCLC cells. (A) Cell death determined in A549 cells at $48 \mathrm{~h}$ after transfection of miR-146a-5p mimic (miR-146a-5p) or miR-NC, stained with propidium iodide (PI) and annexin-FITC. Q1: Live cells; Q2: early apoptotic cells; Q3: late apoptotic cells; Q4: necroptotic cells. (B)

Apoptosis in $\mathrm{H} 1299$ cells at $48 \mathrm{~h}$ after transfection of miR-146a-5p inhibitor or NC inhibitor by FACS as in (A). (C, D) Quantification of the early and late apoptosis of (A, B). (E) Level of NF-kB-p65(pS536), NF-kB-p65, Bax, Bcl-2, cleaved Caspase 3, Caspase 3, cleave PARP and PARP detected by Western blot in A549 cells and H1299 cells at 48 h post transfection of miR-146a-5p mimic or miR-146a-5p inhibitors, respectively. GAPDH or $\beta$-actin used as loading controls. (F). Quantification of pNF-kB-p65(pS536)/NF-kB-p65 ratio as in (E). (G) Quantification of Bax/Bcl2 ratio as in (E). (H) Quantification of cleaved Caspase 3/Caspase 3 ratio as in (E). (I) Quantification of cleaved PARP/PARP ratio as in (E). 
A

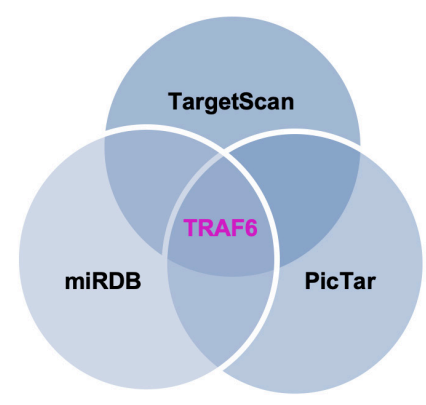

C

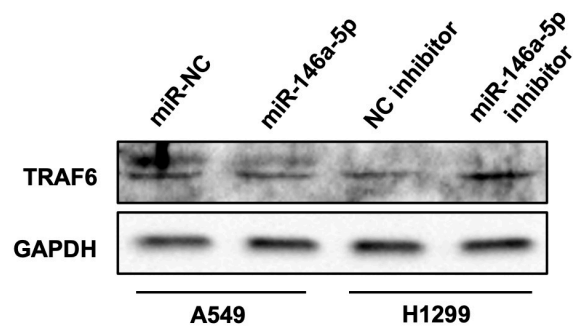

$\mathrm{E}$

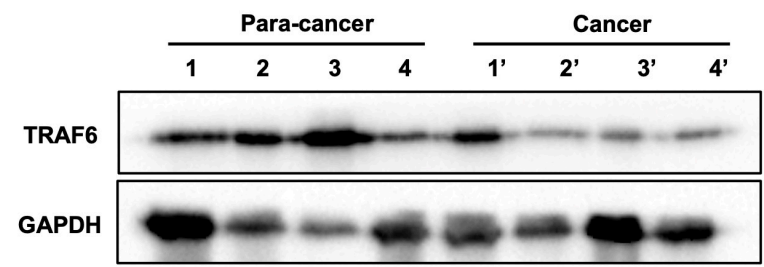

G

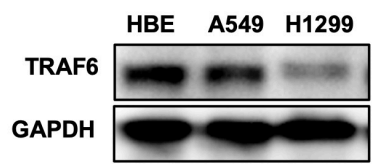

hsa-miR-146a-5p: 3'-UCGGGUACCUUAAGUCAAGAGU-5

TRAF6 3'UTR: 5'-AAUAUCGUGGAAUCUAGUUCUCAGGG-3'

pmirGLO-TRAF6-WT: 5'-CGGGCCCAGAAAGTTGAGTTCTCATTTTC-3' pmirGLO-TRAF6-MUT: 5'-CGGGCCCAGAAAGTTGAGAAGTCATTTTC-3'
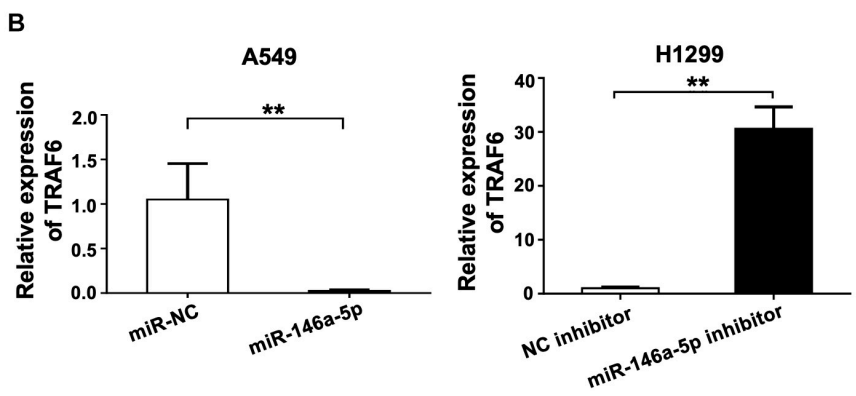

D
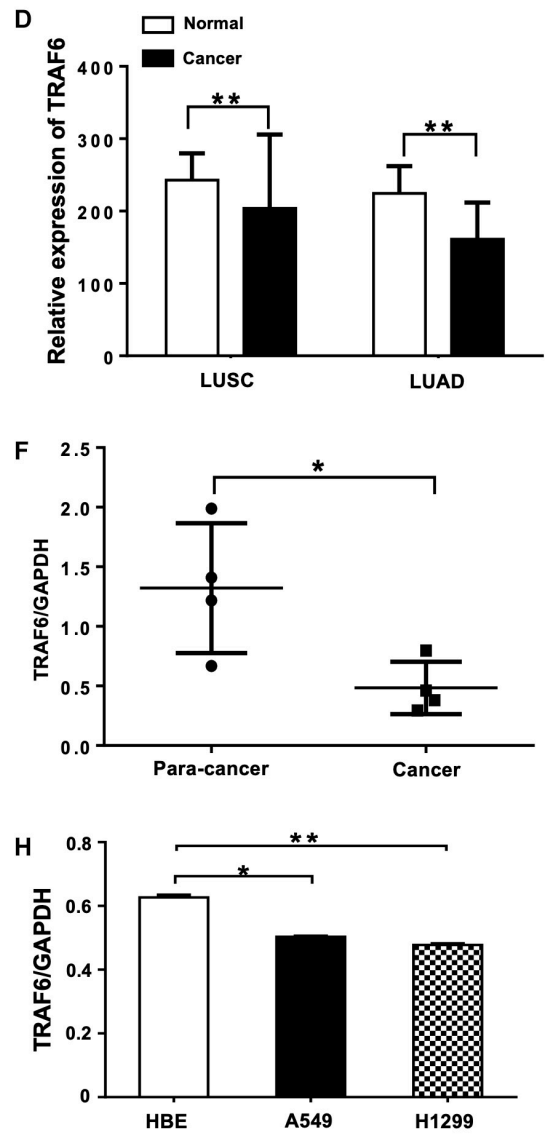

$\mathbf{J}$

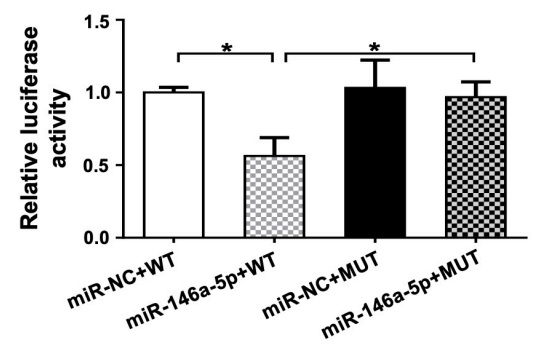

FIGURE 5 | miR-146a-5p directly targets TRAF6 in NSCLC Cells. (A) TRAF6 is one of miR-146a-5p targets predicted by TargetScan, miRDB and PicTar databases. Expression of TRAF6 mRNA (B) and protein (C) in A549 cells or H1299 cells at $24 \mathrm{~h}$ transfection with miR-146a-5p mimic, or miR-146a-5p inhibitor respectively. (D) Expression of TRAF6 from TCGA, LUSC: lung squamous cell carcinoma, LUAD: lung adenocarcinoma. (E) Differential TRAF6 protein levels in NSCLC tissues and the paired adjacent normal tissues. (F) Quantitation of WB data as in (E). (G) TRAF6 levels in two NSCLC cell lines and HBE cell line. (H) Quantitation of WB data as in (G). (I) miR-146a-5p binding site within the 3'UTR of human TRAF6 mRNA predicted by TargetScan. Luciferase reporter construct only contains the 7 bp seed sequence and with the center 3 bp mutated in the mutant construct. (J) The relative luciferase activity in A549 cells co-transfected with miR-146a-5p mimic WT and MUT luciferase reporter ( $24 \mathrm{~h}$ post transfection). 
A

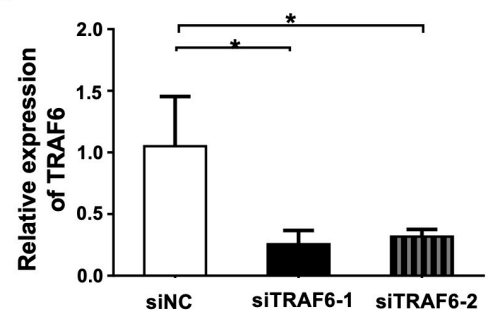

D

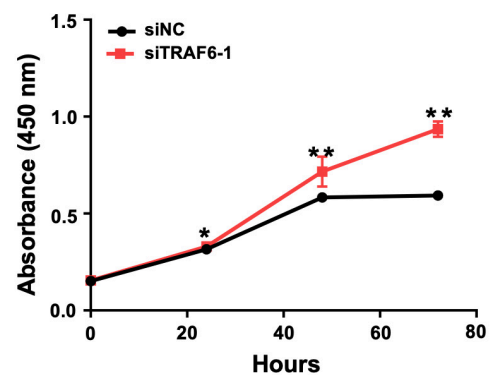

F SiNC SiTRAF6-1

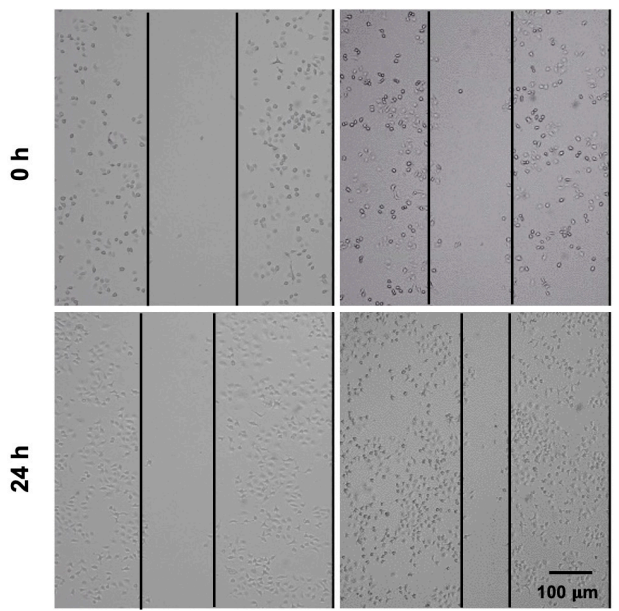

B

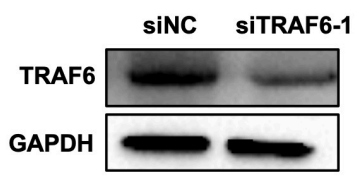

E

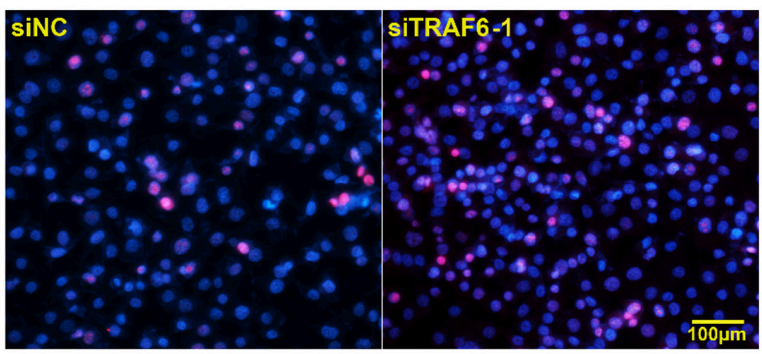

G

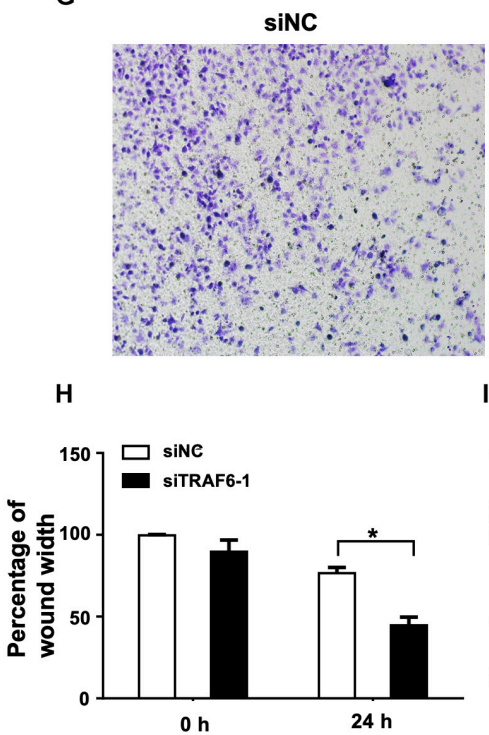

K

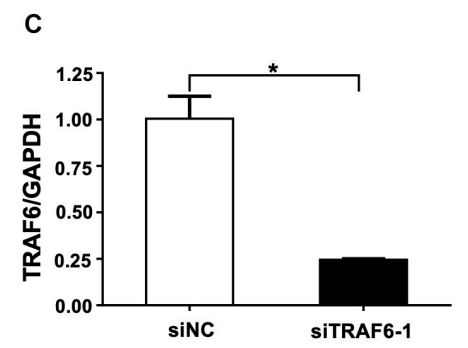

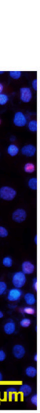

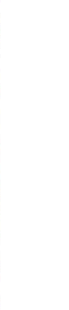


A

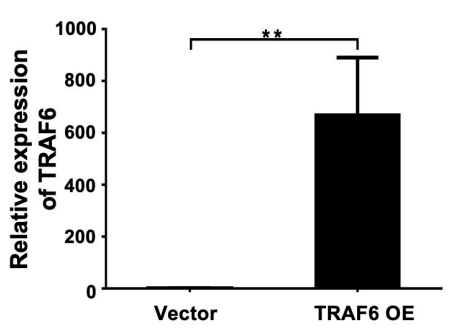

D

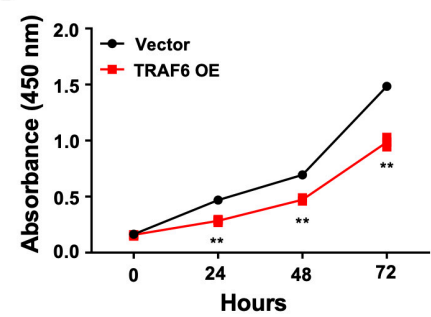

G

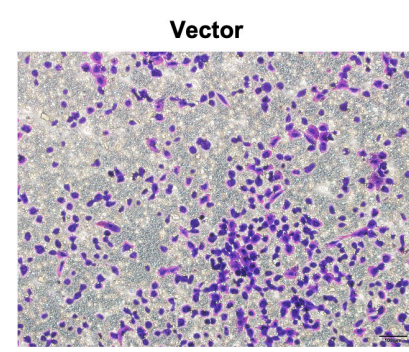

I

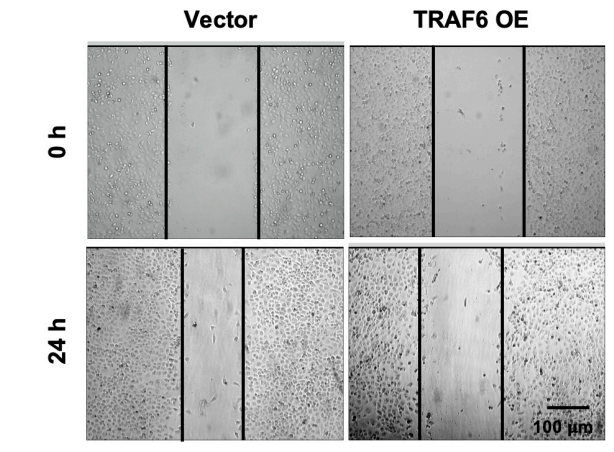

K

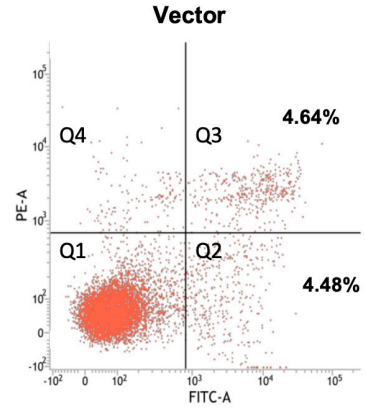

B

E

TRAF6 OE
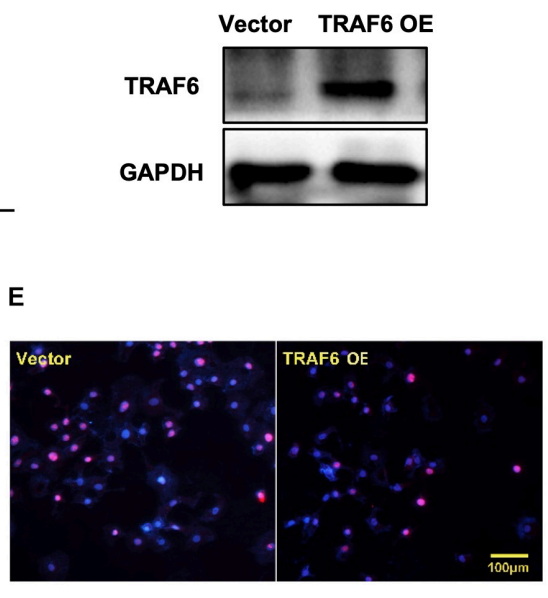

TRAF6 OE
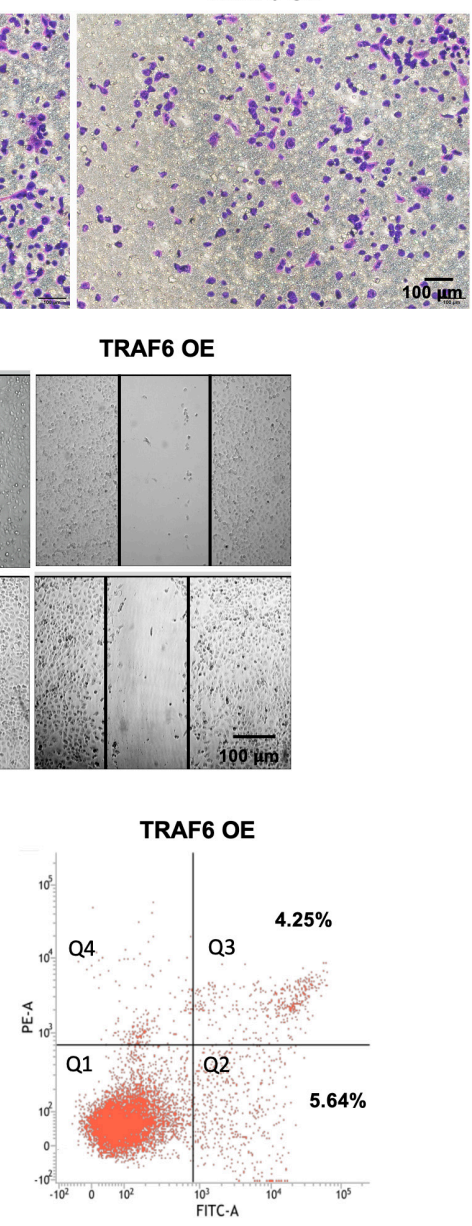

C

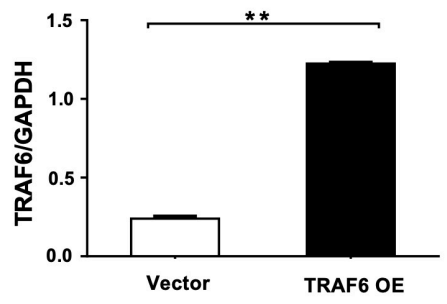

F

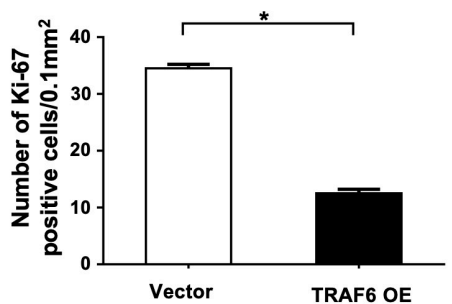

H

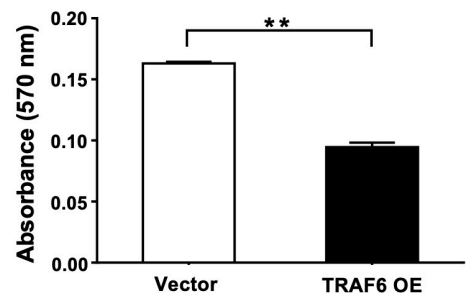

J

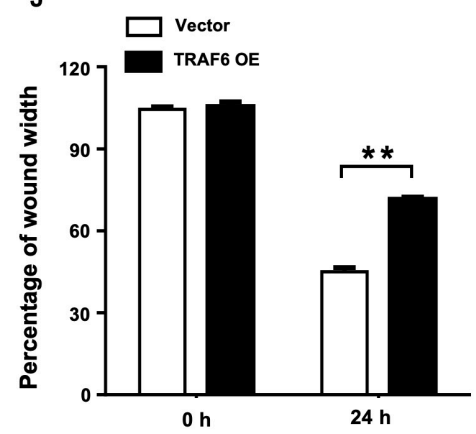

L

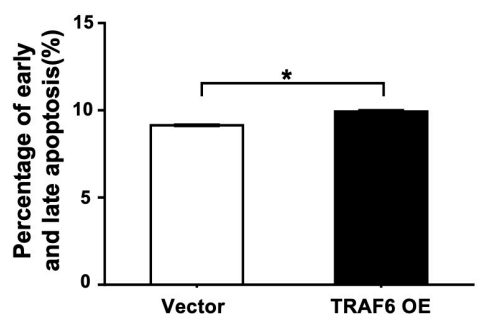

FIGURE 7 | Overexpression of TRAF6 inhibits cell proliferation and migration but promotes apoptosis of NSCLC cells. (A) The expression of TRAF6 examined by qPCR. (B) The protein level of TRAF6. (C) Quantitation of the WB data as in (B). (D) Cell proliferation examined by CCK-8 assay. (E) Cell proliferation measured by Ki-67 staining. (F) Quantification of the Ki-67 staining assay result as in (E). (G) Migration ability detected by transwell assay. (H) Quantification of the transwell assay result as in (G). (I) Migration ability measured by wound-healing assay. (J) Quantification of the wound-healing assay result as in (I). (K) Early (Q2) and late apoptosis (Q3) measured by flow cytometry analysis. (L) Quantification of the FACS data as in (K). All experiments were performed in H1299 cells transfected with TRAF6 overexpression construct for $24 \mathrm{~h}$ except in (K) for $48 \mathrm{~h}$. TRAF6 OE: overexpression vector of TRAF6. 


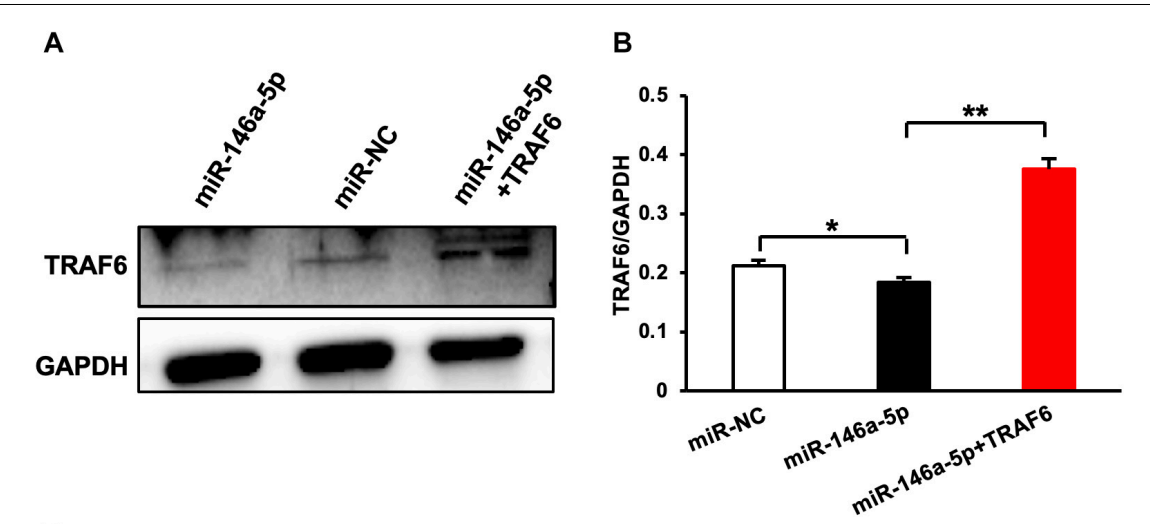

D

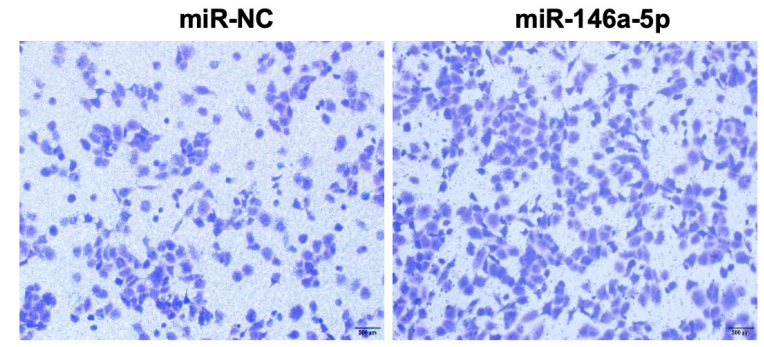

miR-146a-5p+TRAF6

F
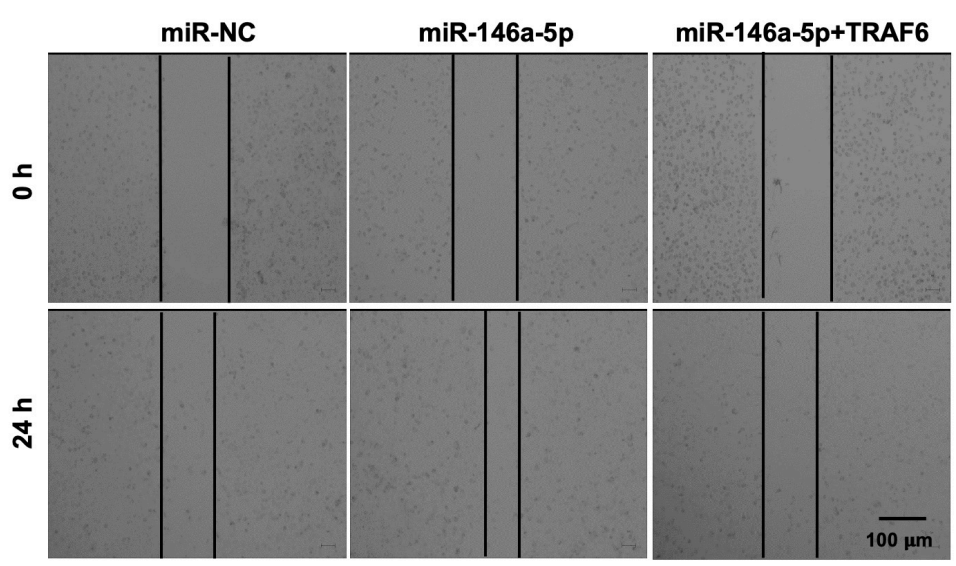

H
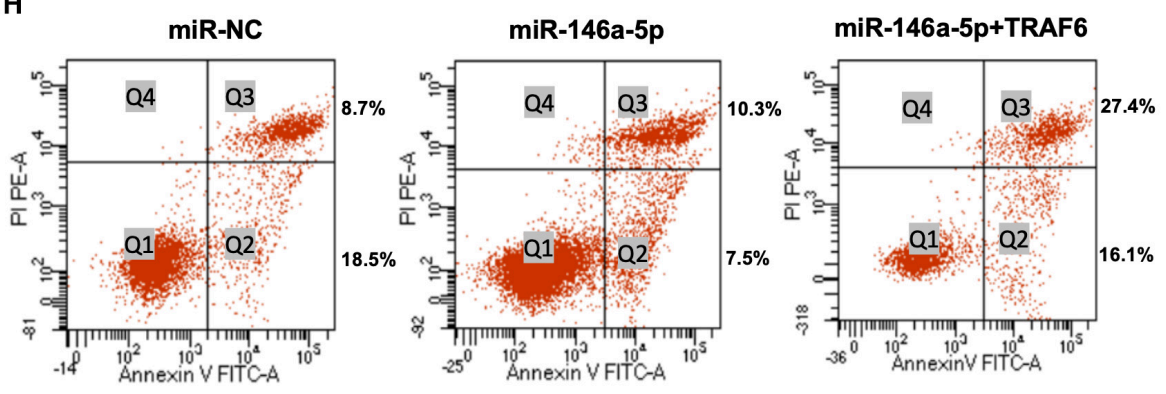

C

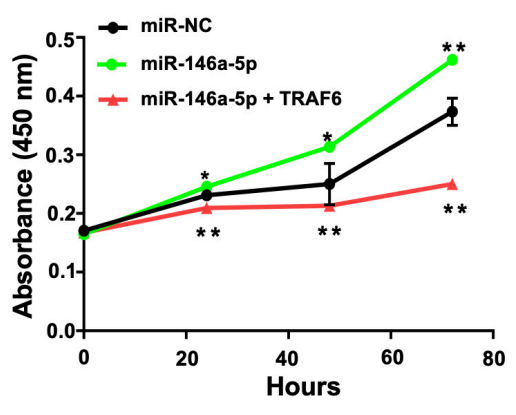

E

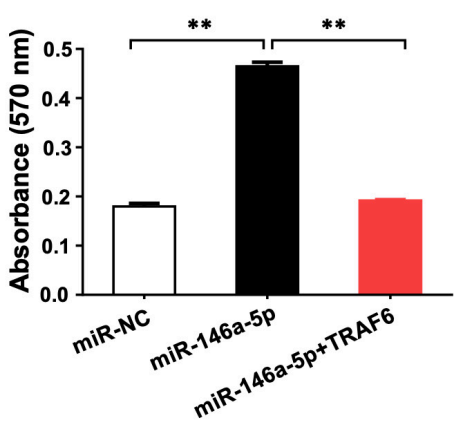

G

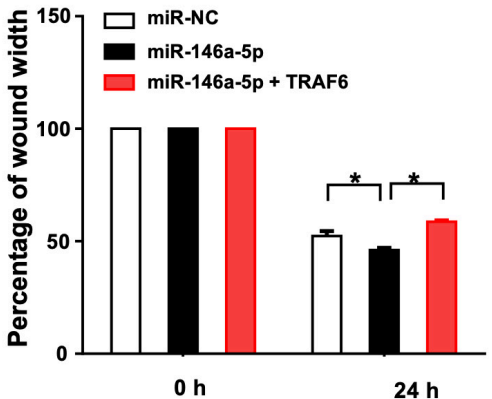

I

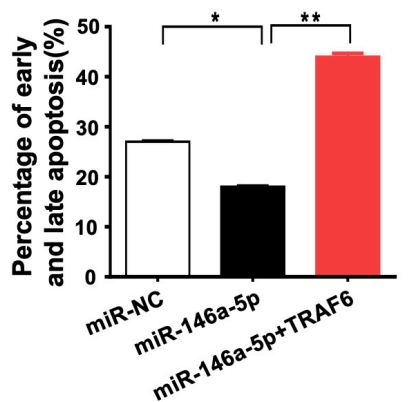

FIGURE 8| Overexpression of TRAF6 reverses the oncogenic effects of miR-146a-5p in NSCLC cells. (A) The protein level of TRAF6. (B) Quantitation of the WB data as in (A). (C) Cell proliferation examined by CCK-8 assay. (D) Migration ability detected by transwell assay. (E) Quantification of the transwell assay result as in (D). (F) Migration ability measured by wound-healing assay. (G) Quantification of the wound-healing assay result as in (F). (H) Early (Q2) and late apoptosis (Q3) measured by flow cytometry analysis. (I) Quantification of the FACS data as in (H). All experiments were performed in H1299 cells. 


\section{DISCUSSION}

By mining the three online miRNA target prediction databases, we found that TRAF6 is one of the overlaps among all intersecting areas, suggesting it is a favorable target of miR-146a-5p. Direct negative regulation of TRAF6 by miR-146a-5p in NSCLC was further validated by dualluciferase reporter assay in the current study. Our Solexa sequencing data demonstrated a significantly elevated serum miR-146a-5p expression in NSCLC patients $(n=36)$, with the concurrence of reduced TRAF6 levels at mRNA and protein levels (Figures 5D-H). By manipulating miR-146a-5p or/and TRAF6 levels in NSCLC cell lines, we found that (1) Overexpression of miR-146a-5p resulted in cell proliferation and reduced TRAF6 expression; (2) Inhibition of miR-146a-5p decreased cancerous cellular behavior, accompanied by higher TRAF6 levels; (3) TRAF6 gene silencing by siRNA recapitulate the outcome of miR-146a-5p over-expression; (4) Forced expression of TRAF6 in conjunction with miR-146a-5p overexpression sufficed to revoke the oncogenic effects of miR-146a-5p. These findings confirm that miR-146a-5p plays an oncogenic role in NSCLC cells via direct suppression of TRAF6.

TRAF6 is indispensable in maintaining a balanced immune function and deletion of TRAF6 in mice is lethal as a result of disrupted development and anatomy of the immune system (Starczynowski et al., 2011; Dou et al., 2018). The miR-146a-5p-TRAF6 axis has been proposed to be a key regulator in immune cell activity, hematopoiesis and malignant transformation, the latter involving chronic inflammation induced by dysregulation of miR-146a (Magilnick et al., 2017). The important roles of TRAF6 in immunity (Dou et al., 2018) and tumorigenesis (Yao et al., 2013) are exhibited in the regulation of NF- $\mathrm{KB}$ signal pathway (Starczynowski et al., 2011; Wang et al., 2012). It has been reported that miR-146a could negatively regulate particulate matter (PM) - induced inflammation via inhibiting the IRAK1/TRAF6 expression and subsequently prevent the nuclear translocation of p65 in BEAS-2B cells (Liu et al., 2018). But the evidence for regulation of TRAF6 by miRNAs outside the immune system is scarce. TRAF6 was reported to be a target of miR146a in papillary carcinoma (Cong et al., 2015) and HCC (Zu et al., 2016).

Except for a few confirmed onco-miRs such as miR$21-5 \mathrm{p}$, the role of many miRNAs in cancers displays an inconsistency that constitutes a great hindrance to their translation into diagnostic or prognostic biomarkers of cancer. As emphasized earlier, the clinical implication of miR-146a-5p in NSCLC is polarized to two opposite conclusions. The discrepancies have multiple origins: tumorintrinsic factors including intratumor heterogeneity, cancer stage and subtypes; cohort factors including tissue/blood sampling, prior treatment, comorbidities, age, sex, and SNP variance; and technical factors including the selection of methods, cell lines and reference genes for RT-PCR normalization, etc. (Condrat et al., 2020). Our data are in agreement with the report from Wang et al. (2015) who found that miR-146a-5p was up-regulated in the serum of NSCLC patients.

However, opposing effects were documented by some other studies. Using different NSCLC cell lines (H358, H1650, and H1975), Chen et al. (2013) found that miR-146a-5p inhibited cell proliferation and induced apoptosis by targeting epidermal growth factor receptor (EGFR) signal pathway. But they did not compare baseline miR-146a-5p levels between NSCLC and normal cells. Besides, they performed transfection of miR146a-5p mimic/inhibitor daily for 10 days (Chen et al., 2013), in contrast to most studies including ours with 24 or $48 \mathrm{~h}$ transfection. Wu et al. (2014) reported that while the stage III cancerous NSCLC tissues or tissues with lymph node metastasis (LNM) displayed lower miR-146a-5p levels than adjacent normal tissues, the majority (over 70\%) of the samples at stage I or without LNM had up-regulated miR-146a-5p. Our Solexa sequencing results showed that miR-146a-5p was increased in stages I, II, and III, but indeed decreased in stage IV. Interestingly, one report showed that miR-146a-5p was a lymphatic metastasis-related miRNA in lung adenocarcinoma (Yan et al., 2017). Li et al. (2016) demonstrated reduced miR146a-5p expression in a few NSCLC cell lines and its suppression of cell proliferation by targeting CCND1 and CCND2. But they also reported only 8 out of 30 clinical cancerous tissues had lowered miR146 levels, which was associated with tumor size $>3 \mathrm{~cm}$ (Li et al., 2016). Considering the involvement of miR-146a-5p in various biological processes and pathways (Li et al., 2016), especially inflammatory response, these reports raise the curiosityof temporal dynamics of miR-146a-5p regulation or a molecular context-dependent dual function of miR-146a$5 \mathrm{p}$, especially when the cancer grade and stage are concerned (Yuan et al., 2020). In our work, restricted by the sample size and insufficient patient characterization, we could not distinguish either the correlation between miR-146a-5p levels and the clinical outcomes or the correlation between miR-146a-5p and TRAF6, although the latter was established in cell lines.

Deep stratification in large cohorts and standardization of detection technologies are essential to substantiate the value of miR-146a-5p in early diagnosis or treatment of NSCLC, very likely in tandem with other NSCLC stage-related miRNAs (Aiso et al., 2018). There also remain many gaps in the mechanistic puzzle. (1) Do the changes in TRAF6 signaling through NF- $\mathrm{KB}$ and AP-1 (Walsh et al., 2015) which directly determine the downstream transcriptional events and form a feedback loop to influence miR-146a$5 \mathrm{p}$ expression? Being transcriptionally regulated by NF- $\mathrm{\kappa B}$, miR-146a-5p itself is an active member participating in the NF-kB-regulated networks controlling inflammatory immune responses and tumorigenesis (Yang and Wang, 2016; Mann et al., 2017). (2) Do the direct overexpression of TRAF6 and the indirect restoration of TRAF6 signaling by miR-146a-5p inhibition instigate the same downstream anti-tumor pathway in NSCLC? In an in vivo miR-146a $\mathrm{a}^{-/-}$ mouse model, lowering TRAF6 expression and thereby dampening NF- $\kappa B$ signaling rescued functions of miR-146a-5p in myeloproliferative and pro-inflammatory regulation, but not in haematopoietic stem cell (HSC) homeostasis 
(Boldin et al., 2011; Magilnick et al., 2017). A plausible scenario is that miR-146a-5p and TRAF6/NF- $\kappa$ B may not form a single linear relationship in NSCLC development. (3) Although a strong correlation between miRNA expression in tissues and body fluids was confirmed in healthy samples, potential discordance between tissue and serum miRNA in the context of cancer is not systematically studied (Cui and Cui, 2020). As miR-146a-5p is critical in maintaining immune homeostasis by braking NF- $\kappa$ Binduced pro-inflammatory reaction, one of the pillar mechanisms promoting malignant transformation, the circulating miR-146a$5 \mathrm{p}$ in NSCLC may not only be secreted from cancer tissues but also reflect the corresponding immune activities against the specific pathological situation (Boldin et al., 2011).

Validation of miRNA as a cancer biomarker could have two different values: early diagnosis, as discussed above, and therapeutic targeting. For instance, miRNAs regulated chemosensitivity in NSCLC treatment (Zhang Y. et al., 2014) and miR-146a promoted the chemosensitivity to cisplatin by targeting cyclin J (Shi et al., 2017) or JNK-2 (Pang et al., 2017) in NSCLC. Targeting EGFR by EGFR- tyrosine kinase inhibitors (TKIs) has opened a new avenue in cancer therapy, as EGFR mutation frequently occurs in a large number of cancers including NSCLC. EGFR is also a target of miR-146, according to miRbase (Nicholson et al., 2001; Griffiths-Jones et al., 2008; Xu et al., 2012). The miR-146 level was found to have a positive predictive value for patients' response to TKIs in NSCLC treatment (Leonetti et al., 2019). Although only certain cancer cell phenotypes were examined, the present work showed that a simple intervention of TRAF6 was sufficient to reverse the malignant behavior of the NSCLC cell lines.

Taken together, despite the discrepancy in the literature, we have placed a diagnostic and therapeutic value on miR146a-5p and its direct target TRAF6 as part of the molecular signature marking NSCLC development. To translate TRAF6dependent miR-146a-5p regulation into clinical application, in a cancerous niche subject to the influence of the immune system and other microenvironmental factors, further analysis of largescale clinical samples is imperative.

\section{REFERENCES}

Aiso, T., Ohtsuka, K., Ueda, M., Karita, S., Yokoyama, T., Takata, S., et al. (2018). Serum levels of candidate microRNA diagnostic markers differ among the stages of non-small-cell lung cancer. Oncol. Lett. 16, 6643-6651. doi: 10.3892/ ol.2018.9464

Bogunia-Kubik, K., Wysoczańska, B., Piątek, D., Iwaszko, M., Ciechomska, M., and Świerkot, J. (2016). Significance of polymorphism and expression of miR$146 \mathrm{a}$ and NFkB1 genetic variants in patients with rheumatoid arthritis. Arch. Immunol. Ther. Exp. 64(Suppl. 1), 131-136. doi: 10.1007/s00005-016-0443-5

Boldin, M. P., Taganov, K. D., Rao, D. S., Yang, L., Zhao, J. L., Kalwani, M., et al. (2011). miR-146a is a significant brake on autoimmunity, myeloproliferation, and cancer in mice. J. Exp. Med. 208, 1189-1201. doi: 10.1084/jem.20101823

Chen, G., Umelo, I. A., Lv, S., Teugels, E., Fostier, K., Kronenberger, P., et al. (2013). miR-146a inhibits cell growth, cell migration and induces apoptosis in non-small cell lung cancer cells. PLoS One 8:e60317. doi: 10.1371/journal.pone. 0060317

Condrat, C. E., Thompson, D. C., Barbu, M. G., Bugnar, O. L., Boboc, A., Cretoiu, D., et al. (2020). miRNAs as biomarkers in disease: latest findings regarding their role in diagnosis and prognosis. Cells 9:276. doi: 10.3390/cells9020276

\section{DATA AVAILABILITY STATEMENT}

All datasets generated for this study are included in the article/Supplementary Material.

\section{ETHICS STATEMENT}

The studies involving human participants were reviewed and approved by Ethics Committee of Second Affiliated Hospital of Dalian Medical University. The patients/participants provided their written informed consent to participate in this study.

\section{AUTHOR CONTRIBUTIONS}

YW, QW, LH, and XL conceived the study, designed the experiments, and wrote the manuscript. XL, BoL, FW, and RL performed the experiments, contributed to the collection of samples, clinical data, and analyzation of data. MZ, YB, JW, NW, $\mathrm{DH}$, and RL also performed some of the experiments, analyzed data, and involved in discussion. ZL, BF, GZ, SW, LZ, JM, YY, and BiL also designed the experiments, involved in discussion, and wrote the manuscript. All authors reviewed the manuscript.

\section{FUNDING}

This work was supported by grants from the National Nature Science Foundation of China (grant nos. 91729302, 81330060, 31871163, and 81600668) and the Provincial Department of Education Research Project (grant no. LQ2017043).

\section{SUPPLEMENTARY MATERIAL}

The Supplementary Material for this article can be found online at: https://www.frontiersin.org/articles/10.3389/fcell.2020.00847/ full\#supplementary-material

Cong, D., He, M., Chen, S., Liu, X., Liu, X., and Sun, H. (2015). Expression profiles of pivotal microRNAs and targets in thyroid papillary carcinoma: an analysis of the cancer genome atlas. Onco Targets Ther. 8, 2271-2277. doi: 10.2147/OTT. S85753

Cui, C., and Cui, Q. (2020). The relationship of human tissue microRNAs with those from body fluids. Sci. Rep. 10:5644. doi: 10.1038/s41598-020-6 2534-6

Di Leva, G., Garofalo, M., and Croce, C. M. (2014). MicroRNAs in cancer. Annu. Rev. Pathol. 9, 287-314. doi: 10.1146/annurev-pathol-012513-104715

Dou, Y., Tian, X., Zhang, J., Wang, Z., and Chen, G. (2018). Roles of TRAF6 in central nervous system. Curr. Neuropharmacol. 16, 1306-1313. doi: 10.2174/ 1570159X16666180412094655

Duan, R., Pak, C., and Jin, P. (2007). Single nucleotide polymorphism associated with mature miR-125a alters the processing of pri-miRNA. Hum. Mol. Genet. 16, 1124-1131. doi: 10.1093/hmg/ddm062

Griffiths-Jones, S., Saini, H. K., van Dongen, S., and Enright, A. J. (2008). miRBase: tools for microRNA genomics. Nucleic Acids Res. 36, D154-D158. doi: 10.1093/ nar/gkm952

Hanahan, D., and Weinberg, R. A. (2000). The hallmarks of cancer. Cell 100, 57-70. doi: 10.1016/s0092-8674(00)81683-9 
Herbst, R. S., Morgensztern, D., and Boshoff, C. (2018). The biology and management of non-small cell lung cancer. Nature 553, 446-454. doi: 10.1038/ nature 25183

Hu, Q., Song, J., Ding, B., Cui, Y., Liang, J., and Han, S. (2018). miR-146a promotes cervical cancer cell viability via targeting IRAK1 and TRAF6. Oncol. Rep. 39, 3015-3024. doi: 10.3892/or.2018.6391

Hung, P. S., Liu, C. J., Chou, C. S., Kao, S. Y., Yang, C. C., Chang, K. W., et al. (2013). miR-146a enhances the oncogenicity of oral carcinoma by concomitant targeting of the IRAK1. TRAF6 and NUMB genes. PLoS One 8:e79926. doi: 10.1371/journal.pone.0079926

Jaeschke, H., Duan, L., Akakpo, J. Y., Farhood, A., and Ramachandran, A. (2018). The role of apoptosis in acetaminophen hepatotoxicity. Food Chem. Toxicol. 118, 709-718. doi: 10.1016/j.fct.2018.06.025

Jalava, S. E., Urbanucci, A., Latonen, L., Waltering, K. K., Sahu, B., Janne, O. A., et al. (2012). Androgen-regulated miR-32 targets BTG2 and is overexpressed in castration-resistant prostate cancer. Oncogene 31, 4460-4471. doi: 10.1038/onc. 2011.624

Jiang, P., Jia, W., Wei, X., Zhang, X., Wang, C., Li, B., et al. (2017). MicroRNA-146a regulates cisplatin-resistance of non-small cell lung cancer cells by targeting NF-kappaB pathway. Int. J. Clin. Exp. Pathol. 10, 11545-11553.

Kumar, S., Keerthana, R., Pazhanimuthu, A., and Perumal, P. (2013). Overexpression of circulating miRNA-21 and miRNA-146a in plasma samples of breast cancer patients. Indian J. Biochem. Biophys. 50, 210-214.

Leonetti, A., Assaraf, Y. G., Veltsista, P. D., El Hassouni, B., Tiseo, M., and Giovannetti, E. (2019). MicroRNAs as a drug resistance mechanism to targeted therapies in EGFR-mutated NSCLC: current implications and future directions. Drug Resist. Updat. 42, 1-11. doi: 10.1016/j.drup.2018.11.002

Lerner, C., Wemmert, S., Bochen, F., Kulas, P., Linxweiler, M., Hasenfus, A., et al. (2016). Characterization of miR-146a and miR-155 in blood, tissue and cell lines of head and neck squamous cell carcinoma patients and their impact on cell proliferation and migration. J. Cancer Res. Clin. Oncol. 142, 757-766. doi: 10.1007/s00432-015-2087-y

Li, T., Li, M., Xu, C., Xu, X., Ding, J., Cheng, L., et al. (2019). miR146a regulates the function of Th17 cell differentiation to modulate cervical cancer cell growth and apoptosis through NFkappaB signaling by targeting TRAF6. Oncol. Rep. 41, 2897-2908. doi: 10.3892/or.2019.7046

Li, Y. L., Wang, J., Zhang, C. Y., Shen, Y. Q., Wang, H. M., Ding, L., et al. (2016). MiR-146a-5p inhibits cell proliferation and cell cycle progression in NSCLC cell lines by targeting CCND1 and CCND2. Oncotarget 7, 59287-59298. doi: 10.18632/oncotarget.11040

Liu, H., Zhang, T., Ye, J., Li, H., Huang, J., Li, X., et al. (2012). TNF receptorassociated factor 6 in advanced non-small cell lung cancer: clinical and prognostic implications. J. Cancer Res. Clin. Oncol. 138, 1853-1863. doi: 10. 1007/s00432-012-1255-6

Liu, L., Wan, C., Zhang, W., Guan, L., Tian, G., Zhang, F., et al. (2018). MiR-146a regulates PM1 -induced inflammation via NF-kappaB signaling pathway in BEAS-2B cells. Environ. Toxicol. 33, 743-751. doi: 10.1002/tox. 22561

Magilnick, N., Reyes, E. Y., Wang, W. L., Vonderfecht, S. L., Gohda, J., Inoue, J. I., et al. (2017). miR-146a-Traf6 regulatory axis controls autoimmunity and myelopoiesis, but is dispensable for hematopoietic stem cell homeostasis and tumor suppression. Proc. Natl. Acad. Sci. U.S.A 114, E7140-E7149. doi: 10.1073/ pnas. 1706833114

Mann, M., Mehta, A., Zhao, J. L., Lee, K., Marinov, G. K., Garcia-Flores, Y., et al. (2017). An NF-kappaB-microRNA regulatory network tunes macrophage inflammatory responses. Nat. Commun. 8:851. doi: 10.1038/s41467-017-0 0972-z

Meng, Q., Liang, C., Hua, J., Zhang, B., Liu, J., Zhang, Y., et al. (2020). A miR$146 a-5 p / T R A F 6 / N F-k B$ p65 axis regulates pancreatic cancer chemoresistance: functional validation and clinical significance. Theranostics 10, 3967-3979. doi: $10.7150 /$ thno.40566

Meng, Q., Zheng, M., Liu, H., Song, C., Zhang, W., Yan, J., et al. (2012). TRAF6 regulates proliferation, apoptosis, and invasion of osteosarcoma cell. Mol. Cell. Biochem. 371, 177-186. doi: 10.1007/s11010-012-1434-4

Meshkat, M., Tanha, H. M., Naeini, M. M., Ghaedi, K., Sanati, M. H., Meshkat, M., et al. (2016). Functional SNP in stem of mir-146a affects Her2 status and breast cancer survival. Cancer Biomark. 17, 213-222. doi: 10.3233/CBM-1 60633
Min, S. K., Jung, S. Y., Kang, H. K., Park, S. A., Lee, J. H., Kim, M. J., et al. (2017). Functional diversity of miR-146a-5p and TRAF6 in normal and oral cancer cells. Int. J. Oncol. 51, 1541-1552. doi: 10.3892/ijo.2017.4124

Nicholson, R. I., Gee, J. M., and Harper, M. E. (2001). EGFR and cancer prognosis. Eur. J. Cancer 37(Suppl. 4), S9-S15. doi: 10.1016/s0959-8049(01)00231-3

Paik, J. H., Jang, J. Y., Jeon, Y. K., Kim, W. Y., Kim, T. M., Heo, D. S., et al. (2011). MicroRNA-146a downregulates NFkappaB activity via targeting TRAF6 and functions as a tumor suppressor having strong prognostic implications in NK/T cell lymphoma. Clin. Cancer Res. 17, 4761-4771. doi: 10.1158/1078-0432.CCR11-0494

Pang, L., Lu, J., Huang, J., Xu, C., Li, H., Yuan, G., et al. (2017). Upregulation of miR-146a increases cisplatin sensitivity of the non-small cell lung cancer A549 cell line by targeting JNK-2. Oncol. Lett. 14, 7745-7752. doi: 10.3892/ol.2017. 7242

Paterson, M. R., and Kriegel, A. J. (2017). MiR-146a/b: a family with shared seeds and different roots. Physiol. Genomics 49, 243-252. doi: 10.1152/ physiolgenomics.00133.2016

Perez-Sanchez, C., Font-Ugalde, P., Ruiz-Limon, P., Lopez-Pedrera, C., CastroVillegas, M. C., Abalos-Aguilera, M. C., et al. (2018). Circulating microRNAs as potential biomarkers of disease activity and structural damage in ankylosing spondylitis patients. Hum. Mol. Genet. 27, 875-890. doi: 10.1093/hmg/ddy008

Polley, E., Kunkel, M., Evans, D., Silvers, T., Delosh, R., Laudeman, J., et al. (2016). Small cell lung cancer screen of oncology drugs, investigational agents, and gene and microRNA expression. J. Natl. Cancer Inst. 108:djw122. doi: 10.1093/jnci/ djw122

Shi, L., Xu, Z., Wu, G., Chen, X., Huang, Y., Wang, Y., et al. (2017). Up-regulation of miR-146a increases the sensitivity of non-small cell lung cancer to DDP by downregulating cyclin J. BMC Cancer 17:138. doi: 10.1186/s12885-017-3132-9

Starczynowski, D. T., Lockwood, W. W., Delehouzee, S., Chari, R., Wegrzyn, J., Fuller, M., et al. (2011). TRAF6 is an amplified oncogene bridging the RAS and NF-kappaB pathways in human lung cancer. J. Clin. Invest. 121, 4095-4105. doi: 10.1172/JCI58818

Thai, T. H., Christiansen, P. A., and Tsokos, G. C. (2010). Is there a link between dysregulated miRNA expression and disease? Discov. Med. 10, 184-194.

Walsh, M. C., Lee, J., and Choi, Y. (2015). Tumor necrosis factor receptorassociated factor 6 (TRAF6) regulation of development, function, and homeostasis of the immune system. Immunol. Rev. 266, 72-92. doi: 10.1111/ imr. 12302

Wang, C., Zhang, W., Zhang, L., Chen, X., Liu, F., Zhang, J., et al. (2016). miR146a-5p mediates epithelial-mesenchymal transition of oesophageal squamous cell carcinoma via targeting Notch2. Br. J. Cancer 115, 1548-1554. doi: 10.1038/ bjc. 2016.367

Wang, L., Fu, C., Cui, Y., Xie, Y., Yuan, Y., Wang, X., et al. (2012). The Ranbinding protein RanBPM can depress the NF-kappaB pathway by interacting with TRAF6. Mol. Cell. Biochem. 359, 83-94. doi: 10.1007/s11010-011-1002-3

Wang, R. J., Zheng, Y. H., Wang, P., and Zhang, J. Z. (2015). Serum miR-125a-5p, miR-145 and miR-146a as diagnostic biomarkers in non-small cell lung cancer. Int. J. Clin. Exp. Pathol. 8, 765-771.

Warth, A., Cortis, J., Soltermann, A., Meister, M., Budczies, J., Stenzinger, A., et al. (2014). Tumour cell proliferation (Ki-67) in non-small cell lung cancer: a critical reappraisal of its prognostic role. Br. J. Cancer 111, 1222-1229. doi: 10.1038/bjc.2014.402

Wu, C., Cao, Y., He, Z., He, J., Hu, C., Duan, H., et al. (2014). Serum levels of miR-19b and miR-146a as prognostic biomarkers for non-small cell lung cancer. Tohoku J. Exp. Med. 232, 85-95. doi: 10.1620/tjem.232.85

Xiao, B., Zhu, E. D., Li, N., Lu, D. S., Li, W., Li, B. S., et al. (2012). Increased miR$146 \mathrm{a}$ in gastric cancer directly targets SMAD4 and is involved in modulating cell proliferation and apoptosis. Oncol. Rep. 27, 559-566. doi: 10.3892/or.2011.1514

Xu, B., Wang, N., Wang, X., Tong, N., Shao, N., Tao, J., et al. (2012). MiR-146a suppresses tumor growth and progression by targeting EGFR pathway and in a p-ERK-dependent manner in castration-resistant prostate cancer. Prostate 72 , 1171-1178. doi: 10.1002/pros.22466

Yan, L., Jiao, D., Hu, H., Wang, J., Tang, X., Chen, J., et al. (2017). Identification of lymph node metastasis-related microRNAs in lung adenocarcinoma and analysis of the underlying mechanisms using a bioinformatics approach. Exp. Biol. Med. 242, 709-717. doi: 10.1177/1535370216677353

Yang, Y., and Wang, J. K. (2016). The functional analysis of MicroRNAs involved in NF-kappaB signaling. Eur. Rev. Med. Pharmacol. Sci. 20, 1764-1774. 
Yao, F., Han, Q., Zhong, C., and Zhao, H. (2013). TRAF6 promoted the tumorigenicity of esophageal squamous cell carcinoma. Tumour Biol. 34, 32013207. doi: 10.1007/s13277-013-0890-0

Yin, Z., Cui, Z., Ren, Y., Xia, L., Li, H., and Zhou, B. (2017). MiR146a polymorphism correlates with lung cancer risk in Chinese nonsmoking females. Oncotarget 8, 2275-2283. doi: 10.18632/oncotarget. 13722

Yuan, F., Zhang, S., Xie, W., Yang, S., Lin, T., and Chen, X. (2020). Effect and mechanism of miR-146a on malignant biological behaviors of lung adenocarcinoma cell line. Oncol. Lett. 19, 3643-3652. doi: 10.3892/ol.2020. 11474

Zhang, X. L., Dang, Y. W., Li, P., Rong, M. H., Hou, X. X., Luo, D. Z., et al. (2014). Expression of tumor necrosis factor receptor-associated factor 6 in lung cancer tissues. Asian Pac. J. Cancer Prev. 15, 10591-10596. doi: 10.7314/apjcp.2014.15. 24.10591

Zhang, Y., Yang, Q., and Wang, S. (2014). MicroRNAs: a new key in lung cancer. Cancer Chemother. Pharmacol. 74, 1105-1111. doi: 10.1007/s00280-0142559-9

Zheng, T., Chou, J., Zhang, F., Liu, Y., Ni, H., Li, X., et al. (2015). CXCR4 3’UTR functions as a ceRNA in promoting metastasis, proliferation and survival of MCF-7 cells by regulating miR-146a activity. Eur. J. Cell. Biol. 94, 458-469. doi: $10.1016 /$ j.ejcb.2015.05.010
Zhou, C., Jiang, C. Q., Zong, Z., Lin, J. C., and Lao, L. F. (2017). miR-146a promotes growth of osteosarcoma cells by targeting ZNRF3/GSK-3 $\beta / \beta$-catenin signaling pathway. Oncotarget 8, 74276-74286. doi: 10.18632/oncotarget.19395

Zu, Y., Yang, Y., Zhu, J., Bo, X., Hou, S., Zhang, B., et al. (2016). MiR-146a suppresses hepatocellular carcinoma by downregulating TRAF6. Am. J. Cancer Res. 6, 2502-2513.

Zugazagoitia, J., Enguita, A. B., Nuñez, J. A., Iglesias, L., and Ponce, S. (2014). The new IASLC/ATS/ERS lung adenocarcinoma classification from a clinical perspective: current concepts and future prospects. J. Thorac. Dis. 6(Suppl. 5), S526-S536. doi: 10.3978/j.issn.2072-1439.2014.01.27

Conflict of Interest: The authors declare that the research was conducted in the absence of any commercial or financial relationships that could be construed as a potential conflict of interest.

Copyright (c) $2020 \mathrm{Liu}, \mathrm{Liu}, \mathrm{Li}$, Wang, Wang, Zhang, Bai, Wu, Liu, Han, Li, Feng, Zhou, Wang, Zeng, Miao, Yao, Liang, Huang, Wang and Wu. This is an open-access article distributed under the terms of the Creative Commons Attribution License (CC BY). The use, distribution or reproduction in other forums is permitted, provided the original author(s) and the copyright owner(s) are credited and that the original publication in this journal is cited, in accordance with accepted academic practice. No use, distribution or reproduction is permitted which does not comply with these terms. 